TRANSACTIONS OF THE

AMERICAN MATHEMATICAL SOCIETY

Volume 350, Number 3, March 1998, Pages 1143-1166

S 0002-9947(98)02115-1

\title{
DYADIC EQUIVALENCE TO COMPLETELY POSITIVE ENTROPY
}

\author{
ADAM FIELDSTEEL AND J. ROBERTO HASFURA-BUENAGA
}

\begin{abstract}
We show that every free ergodic action of $\bigoplus_{1}^{\infty} \mathbb{Z}_{2}$ of positive en-
\end{abstract} tropy is dyadically equivalent to an action with completely positive entropy.

\section{IntroduCtion: $\Gamma$ ACTIONS AND DYADIC EQUIVALENCE}

Let $\Gamma$ denote the group

$$
\bigoplus_{1}^{\infty} \mathbb{Z}_{2}=\left\{g \in\{0,1\}^{\mathbb{N}}: g(i)=1 \text { for only finitely many } i\right\},
$$

under coordinate-wise addition. By an action $T$ of $\Gamma$ on a Lebesgue probability space, $(X, \mathcal{F}, \mu)$, we mean a group homomorphism $T$ from $\Gamma$ to the group Aut $(X, \mathcal{F}, \mu)$ of $\mu$-preserving automorphisms of $X$. We denote the image of $g$ under $T$ by $T^{g}$, and for each $A \subset \Gamma$, we denote the set $\left\{T^{g} x: g \in A\right\}$ by $T^{A}(x)$, or more simply, by $A(x)$, when the action $T$ is understood. An action $T$ of $\Gamma$ on $(X, \mathcal{F}, \mu)$ is called free if, for all $g \in \Gamma$, other than the identity, $\mu\left\{x \in X: T^{g}(x)=x\right\}=0$. A $\Gamma$-action $T$ is called ergodic if every $T$-invariant set has measure 0 or 1 .

Two $\Gamma$-actions $T_{1}$ and $T_{2}$ on spaces $\left(X_{i}, \mathcal{F}_{i}, \mu_{i}\right), i=1,2$, are isomorphic if there is a measure theoretic isomorphism $\phi: X_{1} \rightarrow X_{2}$ such that for almost all $x$ and for all $g \in \Gamma$,

$$
\phi T_{1}^{g}(x)=T_{2}^{g} \phi(x) .
$$

$T_{1}$ and $T_{2}$ are orbit equivalent if there is a measure theoretic isomorphism $\phi: X_{1} \rightarrow$ $X_{2}$ such that for almost all $x$,

$$
\phi T_{1}^{\Gamma}(x)=T_{2}^{\Gamma}(\phi(x)) .
$$

In this paper we study a notion of equivalence of $\Gamma$-actions that is weaker than isomorphism but stronger than orbit equivalence. Let

$$
\Gamma_{n}=\left\{g \in\{0,1\}^{\mathbb{N}}: g(i)=0 \text { for all } i>n\right\},
$$

a subgroup of $\Gamma$ of order $2^{n}$. We say that $\Gamma$-actions $T_{1}$ and $T_{2}$ on spaces $\left(X_{i}, \mathcal{F}_{i}, \mu_{i}\right)$, $i=1,2$, are dyadically equivalent if there is a measure theoretic isomorphism $\phi$ : $X_{1} \rightarrow X_{2}$ such that for almost all $x$ and for all $n \in \mathbb{N}$,

$$
\phi T_{1}^{\Gamma_{n}}(x)=T_{2}^{\Gamma_{n}}(\phi(x)) .
$$

Received by the editors March 6, 1996.

1991 Mathematics Subject Classification. Primary 28D15, 28D20; Secondary 58F11.

Key words and phrases. Ergodic theory, measurable dynamics, orbit equivalence. 
The study of this notion of equivalence has its origins in some questions of a less evidently dynamical nature, which were investigated by Stepin [12] and Vershik [14][15]. Suppose $\left\{\mathcal{F}_{n}\right\}_{n=0}^{\infty}$ is a decreasing sequence of $\sigma$-algebras in a Lebesgue probability space $\left(X, \mathcal{F}_{0}, \mu\right)$, such that each of the $\sigma$-algebras $\mathcal{F}_{n}$ is a two point extension of the next, and the involution that maps each point in $\left(X, \mathcal{F}_{n}, \mu\right)$ to the other member of its equivalence class, $\bmod \mathcal{F}_{n+1}$, is measure preserving. Suppose, further, that $\bigcap_{n} \mathcal{F}_{n}$ consists only of sets of measure 0 and 1. Stepin and Vershik asked whether any two such structures (called ergodic dyadic sequences) must be isomorphic, in the sense that there is a measure theoretic isomorphism carrying one sequence to the other.

Every free action $T$ of $\Gamma$ gives rise to a dyadic sequence $\left\{\mathcal{F}_{n}\right\}_{n=0}^{\infty}$, by setting

$$
\mathcal{F}_{n}=\left\{B \in F: \forall g \in \Gamma_{n}, T^{g} B=B\right\} .
$$

On the other hand, for every dyadic sequence, one can introduce a (necessarily free) action $T$ of $\Gamma$ which gives rise to it in this way. However, the $\Gamma$-action that does this is not unique. More precisely, $\Gamma$-actions $T_{1}$ and $T_{2}$ give rise to isomorphic dyadic sequences if and only if $T_{1}$ and $T_{2}$ are dyadically equivalent. Stepin and Vershik showed, among other things, that $\Gamma$-actions that are dyadically equivalent must have the same entropy, and consequently there are many isomorphism classes of ergodic dyadic sequences. They also gave examples of dyadically equivalent but non-isomorphic ergodic $\Gamma$-actions. More recently, Hasfura-Buenaga showed [2] that the dyadic equivalence class of every free ergodic $\Gamma$-action contains a mixing action. In this paper we will prove a result of a similar type: the dyadic equivalence class of every free ergodic $\Gamma$-action of positive entropy contains an action of completely positive entropy.

Dyadic equivalence may be viewed as an analogue, in the setting of $\Gamma$-actions, of the notion of Kakutani equivalence for $\mathbb{Z}$-actions. Indeed, a very general theory has been developed by Rudolph [6], and is being extended by Kammeyer and Rudolph [4], which gives a unified treatment of a large class of such notions of restricted orbit equivalence, for various acting groups. Their work describes the entropy associated with each example of restricted orbit equivalence, and identifies for each a special class of actions that are characterized up to equivalence by their entropy. We anticipate that dyadic equivalence will be an example to which their work applies.

The first result of the type we will prove here was obtained by Ornstein and Smorodinsky with respect to Kakutani equivalence of $\mathbb{Z}$-actions [7]. Their argument was adapted in [1] to give the corresponding result for $\mathbb{Z}^{n}$-actions, with repect to several notions of equivalence. Here we will again follow their methods to obtain our result. The proof (as in each of these settings) is a construction, and has two main ingredients. We need a family of permutations of orbits (which, by virtue of the freeness of the action, are permutations of the acting group), which can be sequentially applied to produce the desired equivalent action, and we need special factors of the action that will specify the portions of the orbits to be permuted and the permutations to be applied. The first will be easy to describe. The second relies upon significant portions of the relative Bernoulli theory. Since, to our knowledge, no development of the relative Bernoulli theory has yet been given for actions of $\Gamma$, we will give one here.

We begin by collecting the basic facts about $\Gamma$-actions that we will need in our arguments. 
We have the following fact, which can be proved by standard methods.

Rokhlin Lemma. Let $T$ be a free ergodic $\Gamma$-action on $(X, \mathcal{F}, \mu)$. For all (finite) partitions $P$ and all $n \in \mathbb{N}$, there is a set $D$ forming the base of a $\Gamma_{n}-$ Rokhlin tower $\left\{T^{g} D\right\}_{g \in \Gamma_{n}}$ for $T$ (by which we mean the sets $T^{g} D$ are pairwise disjoint), such that $\mu\left(\bigcup_{g \in \Gamma_{n}} T^{g} D\right)=1$ and $\operatorname{dist}\left(P_{D}\right)=\operatorname{dist}(P)$. (See section 2 for notation).

We will also use the ergodic theorem, which we state here only for the ergodic case:

Ergodic theorem. Let $T$ be an ergodic $\Gamma$-action on $(X, \mathcal{F}, \mu)$. For all $f \in L^{1}(\mu)$,

$$
\frac{1}{2^{n}} \sum_{g \in \Gamma_{n}} f\left(T^{g}(x)\right) \rightarrow \int_{X} f d \mu \quad \text { a.e. }
$$

This can be obtained as a special case of the ergodic theorem proved in [9].

\section{Entropy of $\Gamma-$ Processes}

2.1. Definitions and basic properties of entropy. By a $\Gamma$-process, we mean a family $\left\{X_{g}\right\}_{g \in \Gamma}$ of random variables indexed by $\Gamma$, whose joint distributions are invariant under translation by elements of $\Gamma$. We will always assume that our processes have a finite state space. Equivalently, a $\Gamma$-process is a pair $(T, P)$ consisting of a $\Gamma$-action $T$ and a finite partition $P=\left\{P_{i}\right\}_{i=1}^{k}$ of the space on which it acts. Given a $\Gamma$-process $(T, P)$, and a set $A \subset \Gamma$, we let $(T, P)_{A}$ denote $\bigvee_{g \in A} T^{-g} P$. The process $(T, P)$ is called ergodic if $T$ restricted to $(T, P)_{\Gamma}$ is ergodic. A finite code of a process $(T, P)$ is a map $\pi: P^{A} \rightarrow S$, where $A \subset \Gamma$ is a finite set. Such a code yields a $(T, P)_{A}$ - measurable partition $\pi(T, P)=\left\{\pi^{-1}(s)\right\}_{s \in S}$. We write $Q \stackrel{\pi, \varepsilon}{\complement}(T, P)_{A}$ if $|Q-\pi(T, P)|<\varepsilon$.

We write $\operatorname{dist}(X)$ to denote the distribution of a variable $X$ and $\operatorname{dist}_{B} X$ to denote the conditional distribution of $X$ on a set $B$ of positive measure. If $B$ is a finite set, $\operatorname{dist}_{B} X$ denotes the distribution of $X$ as measured by the normalized counting measure on $B$.

For each $x \in X$, we let $(T, P)_{A}(x)$ denote either the $(T, P, A)$-name of $x$, which is the function from $A$ to $P$ such that $(T, P)_{A}(x)(g)=P_{i}$ if and only if $T^{g}(x) \in P_{i}$, or the set of points having that name. The context will make clear which meaning is intended. We say two $\left(T, \Gamma_{n}\right)$-names $c$ and $d$ are $\Gamma_{n}$-rotations of one another if there exists $g^{\prime} \in \Gamma_{n}$ such that for all $g \in \Gamma_{n}, c\left(g g^{\prime}\right)=d(g)$. In other words, if $c=(T, P)_{\Gamma_{n}}(x)$, then $d=(T, P)_{\Gamma_{n}}\left(T^{g^{\prime}} x\right)$, for some $g^{\prime} \in \Gamma_{n}$. In this case, we will write $d=g^{\prime} c$. Similar terminology and notation will be used in the case of names that are defined on proper subsets of the $\Gamma_{n}$.

We define the entropy of a $\Gamma$-process $(T, P)$ to be

$$
h(T, P)=\lim _{n \rightarrow \infty} \frac{1}{2^{n}} H\left((T, P)_{\Gamma_{n}}\right),
$$

where $H$ is the usual entropy function on finite partitions:

$$
H(Q)=\sum_{i=1}^{i=k} \mu\left(Q_{i}\right) \log \left(\mu\left(Q_{i}\right)\right),
$$


for $Q=\left\{Q_{i}\right\}_{i=1}^{k}$. This limit can be shown to exist by standard arguments, but in fact, we know from a theorem of Ornstein and Weiss [9] that if $(T, P)$ is ergodic, the information function

$$
-\frac{1}{2^{n}} \log \left(\mu\left((T, P)_{\Gamma_{n}}(x)\right)\right)
$$

converges almost everywhere to a constant $h$, which must then coincide with the above limit. It follows that

$$
h(T, P)=\inf \left\{\begin{array}{c}
h:(\forall \varepsilon>0)\left(\exists n_{0}\right)\left(\forall n \geq n_{0}\right) \text { some collection } \mathcal{C} \\
\text { consisting of fewer than } 2^{2^{n}(h+\varepsilon)} \text { atoms } \\
\text { of }(T, P)_{\Gamma_{n}} \text { satisfies } \mu(\bigcup \mathcal{C})>1-\varepsilon
\end{array}\right\} .
$$

We will make use of several basic properties of the entropy, which we list here. They are all analogous to properties of the entropy of $\mathbb{Z}$-processes, and are proved in similar fashion.

Lemma 1. For all $\Gamma$-actions $T$ and finite partitions $P$ and $Q$,

1. $h(T, P) \leq H(P)$,

2. $h(T, P \vee Q) \leq h(T, P)+h(T, Q)$.

Lemma 2. For all $\Gamma$-actions $T$, finite partitions $P$, and $n \in \mathbb{N}, h\left(T,(T, P)_{\Gamma_{n}}\right)=$ $h(T, P)$.

(In fact, note that for all $m>n$, the partitions $\left(T,(T, P)_{\Gamma_{n}}\right)_{\Gamma_{m}}$ and $(T, P)_{\Gamma_{m}}$ coincide.)

Lemma 3. For all $\varepsilon>0$ and $k \in \mathbb{N}$, there is a $\delta>0$ such that for all $\Gamma$-actions $T$ and all $k$-set partitions $P$ and $Q$ satisfying $|P-Q|<\delta$, we get

$$
|h(T, P)-h(T, Q)|<\varepsilon \text {. }
$$

Lemma 4. For all $\Gamma$-actions $T$, and finite partitions $P$ and $Q$, if $Q \subset(T, P)_{\Gamma}$, then $h(T, Q) \leq h(T, P)$.

Lemma 5. For all $\varepsilon>0$, there exists $\delta>0$, depending on $\varepsilon$ and $\# Q$, such that, for all $\Gamma$-actions $T$ and finite partitions $P$ and $Q$, if $Q \stackrel{\delta}{\subset}(T, P)_{\Gamma}$, then $h(T, P)>$ $h(T, Q)-\varepsilon$.

Lemma 6. If $(T, P \vee Q)$ is an ergodic $\Gamma$-process with $T$ acting freely on $(P \vee Q)_{\Gamma}$, and $h(T, P \vee Q)>h(T, Q)$, then for all $\varepsilon>0$, there is a partition $P^{\prime} \subset(T, P \vee Q)_{\Gamma}$ such that $\left|P^{\prime}-P\right|<\varepsilon$ and $h\left(T, P^{\prime} \vee Q\right)<h(T, P \vee Q)$.

The proof of the corresponding lemma for $\mathbb{Z}$-actions (see [7]) can be adapted to this setting.

\subsection{Completely positive entropy.}

Definition 1. A $\Gamma$-action $T$ has completely positive entropy if for all non-trivial partitions $P, h(T, P)>0$.

We should note that $\Gamma$-actions of completely positive entropy exist. It is easy to verify, for example, that the $\Gamma$-action arising from an independent $\Gamma$-process is such an action.

We write $Q \perp^{\varepsilon} P$ to mean that on each atom in a set of $P$ atoms whose measure is greater than $1-\varepsilon$, the conditional distribution of $Q$ is within $\varepsilon$ of dist $Q$. 
Definition 2. A $\Gamma$-process $(T, P)$ is $K$-mixing, if for every $k \in \mathbb{N}$ and every $\varepsilon>0$ there exists an $n \in \mathbb{N}$ such that for all $m \geq n,(T, P)_{\Gamma_{k}} \perp^{\varepsilon}(T, P)_{\Gamma_{m} \backslash \Gamma_{n}}$. (The notation $\Gamma_{m} \backslash \Gamma_{n}$ denotes the set difference: $\left.\left\{g \in \Gamma_{m}: g \notin \Gamma_{n}\right\}\right)$.

Definition 3. A $\Gamma$-action $T$ is $K$-mixing if for some increasing sequence of partitions $P_{i}$ such that $\bigvee_{i=1}^{\infty} P_{i}=\mathcal{F}$, and for each $i,\left(T, P_{i}\right)$ is $K$-mixing.

Lemma 7. If a $\Gamma$-action $T$ is $K$-mixing, then $T$ has completely positive entropy.

Proof. We adapt the proof given of lemma 2 in [1] as follows. Fix a partition $P$ and choose $\varepsilon>0$ so that if partitions $Q$ and $Q^{\prime}$ with \#P elements satisfy $\left|Q-Q^{\prime}\right|<\varepsilon$, then for every $\Gamma$-action $S,\left|h(S, Q)-h\left(S, Q^{\prime}\right)\right|<\frac{H(P)}{2}$. Choose a partition $P_{i}$ from the generating sequence and $P^{\prime} \subset P_{i}$ such that $\left|P-P^{\prime}\right|<\varepsilon$ and so that $H\left(P^{\prime}\right)>\frac{H(P)}{2}$. For each $n \in \mathbb{N}$, let $T^{(n)}$ denote the restriction of $T$ to the subgroup $\Gamma^{n}=\{g \in \Gamma: g(i)=0$ for $i \leq n\}$. This can again be regarded as an action of $\Gamma$, and if $n$ is taken to be sufficiently large, we have $h\left(T^{(n)}, P^{\prime}\right)>\frac{H(P)}{2}$, and consequently $h\left(T^{(n)}, P\right)>0$. It follows that $h(T, P)>0$, as desired.

Note that in the proof of lemma 7, we didn't use the full strength of the hypotheses. We formulate this observation as an additional lemma.

Definition 4. A $\Gamma$-action $T$ is $K$-mixing at the origin if for some increasing sequence of partitions $P_{i}$ such that $\bigvee_{i=1}^{\infty} P_{i}=\mathcal{F}$, and for each $i$ and each $\varepsilon>0$, there exists an $n \in \mathbb{N}$ such that for all $m>n, P_{i} \perp^{\varepsilon}\left(T, P_{i}\right)_{\Gamma_{m} \backslash \Gamma_{n}}$.

Lemma 8. If a $\Gamma$-action $T$ is $K$-mixing at the origin, then $T$ has completely positive entropy.

In the proof of our main theorem (Theorem 5) we will use this property, rather than $K$-mixing, to obtain completely positive entropy. This is purely a matter of convenience, since the proof can be modified to achieve $K$-mixing as well.

We remark that for all ergodic $\Gamma$-actions $T$, even if $T$ is $K$-mixing, it is not the case that for every partition $Q,(T, Q)$ is $K$-mixing. To see this, we introduce a certain tail field associated with a $\Gamma$-process.

Definition 5. For each $n \in \mathbb{N}$, and partition $P$, we set $\mathcal{T}_{n, P}=(T, P)_{\Gamma \backslash \Gamma_{n}}$, and $\mathcal{T}_{P}=\bigcap_{n=1}^{\infty} \mathcal{T}_{n, P}$.

Standard arguments show that $(T, P)$ is $K$-mixing if and only if $\mathcal{T}_{P}$ is trivial. However, every ergodic $\Gamma$-process $(T, Q)$ admits a generator $P$ (that is, a partition $P$ with $\left.(T, P)_{\Gamma}=(T, Q)_{\Gamma}\right)$ such that $\mathcal{T}_{P}=(T, Q)_{\Gamma}$. This can be proved using the same sort of argument that Ornstein and Weiss use to construct bilaterally deterministic generators for $\mathbb{Z}$-actions [8]. Thus, in particular, a $\Gamma$-process $(T, Q)$ of completely positive entropy (i.e. such that $h(T, R)>0$ for all partitions $R \subset$ $\left.(T, P)_{\Gamma}\right)$ will admit partitions $P$ such that $(T, P)$ is not $K$-mixing.

Definition 6. For $\Gamma$-actions $T$, we set $h(T)=\sup _{\text {finite } P} h(T, P)$.

\section{BASIC BERNOUlLI FACTS}

3.1. Special Bernoulli processes. In order to direct the orbit changes that produce a $\Gamma$-action with completely positive entropy, we will make use of a special class of $\Gamma$-processes that are factors of independent $\Gamma$-processes. For each $n \in \mathbb{N}$ 
we define a $\Gamma$-process $X^{(n)}=\left\{X_{k}^{(n)}\right\}_{k \in \Gamma}$, which we refer to as the $(n)$-Bernoulli process. For given $n$, the state space of the process will be $\Gamma_{n}$, and the distribution of $X^{(n)}$ is the measure $\lambda$ on $\left(\Gamma_{n}\right)^{\Gamma}$ such that

1. on each coset $g \Gamma_{n}$ of $\Gamma_{n}$, the marginal $\lambda_{g \Gamma_{n}}$ of $\lambda$ is uniformly distributed on the constant functions in $\left(\Gamma_{n}\right)^{g \Gamma_{n}}$, and

2. for each $k>n$, the marginal $\lambda_{\Gamma_{k}}$ of $\lambda$ is the direct product $\prod_{h} \lambda_{h \Gamma_{n}}$, where the product is extended over a family of representatives of the cosets of $\Gamma_{n}$ in $\Gamma_{k}$.

Informally, we can describe this process by saying that it assigns a constant element of $\Gamma_{n}$ to each coset of $\Gamma_{n}$ in $\Gamma$ with uniform distribution on $\Gamma_{n}$, and these assignments on distinct cosets of $\Gamma_{n}$ are made independently.

The process $X^{(n)}$ is a factor of the full $\Gamma-2$-shift, by which we mean the independent $\Gamma$-process $Y=\left\{Y_{k}\right\}_{k \in \Gamma}$ on $\{0,1\}^{\Gamma}$ which, for each $m$, gives each element of $\{0,1\}^{\Gamma_{m}}$ equal probability. To exhibit $X^{(n)}$ as a factor, we form a partition $P=\left\{P_{i}\right\}_{i=1}^{2^{n}}$ of $\{0,1\}^{\Gamma_{n}}$ into $2^{n}$ sets, each of cardinality $2^{2^{n}-n}$, and each of which is closed under taking $\Gamma_{n}$-rotations. That this can be done can be seen by the following argument.

First note that $\Gamma_{n}$ acts on $\{0,1\}^{\Gamma_{n}}$ by rotation, so each orbit under this action has cardinality $2^{k}$, for some $k=0,1,2, \ldots, n$. Picture each set $P_{i}$ as a file (empty at first) of capacity $2^{2^{n}-n}$, divided into $2^{2^{n}-2 n}$ drawers of capacity $2^{n}$. We must place the orbits of this action of $\Gamma_{n}$ into these files, so that each orbit is placed in a single file. Begin by placing (whole) orbits of cardinality $2^{n}$ into the drawers of $P_{1}$, filling as many as possible and continuing to fill $P_{2}, P_{3}$, and so on, as far as possible. When the orbits of size $2^{n}$ are exhausted, continue filling drawers with orbits of size $2^{n-1}$, and so on. Continuing in this fashion, we note that at each stage, the orbits remaining to be placed in drawers are of a cardinality that divides the cardinality of the spaces in drawers that remain to be filled, so there is no obstruction to filling all the $P_{i}$.

Now let $\phi$ be a bijection from $P$ to $\Gamma_{n}$. For each $g \in \Gamma$, and for each $\omega$ in the underlying probability space, we have a map $\varsigma_{Y, g}(\omega) \in\{0,1\}^{\Gamma_{n}}$ defined by setting $\varsigma_{Y, g}(\omega)(h)=Y_{h g}(\omega)$. Now we set, for each $g$ and $\omega, \widetilde{X}_{g}^{(n)}(\omega)=\phi\left(\varsigma_{Y, g}(\omega)\right)$. The process $\widetilde{X}^{(n)}=\left\{\widetilde{X}_{g}^{(n)}\right\}_{g \in \Gamma}$ has the same distribution as $X^{(n)}$.

Informally, we obtain $X^{(n)}$ from the 2 -shift $Y$ by a $\Gamma$-invariant code of "code length" $\Gamma_{n}$.

We point out that the process $X^{(n)}$ is not free. In other words, the $\Gamma$-shift on the extension of $X^{(n)}$ in the sequence space $\left(\Gamma_{n}\right)^{\Gamma}$ does not act freely. Indeed, every element of $\Gamma_{n}$ acts like the identity. Thus, although it is a factor of the $2-$ shift, it is not isomorphic to an independent $\Gamma$-process.

We note further that $\lim _{n \rightarrow \infty} h\left(X^{(n)}\right)=0$. Indeed for each $k>n$, there are $\left(2^{n}\right)^{2^{k-n}} \Gamma_{k}$-names, and each is given the same probability. Therefore,

$$
\frac{1}{2^{k}} H\left(\left\{X_{g}^{(n)}\right\}_{g \in \Gamma_{k}}\right)=\frac{1}{2^{k}} n\left(2^{k-n}\right)=\frac{n}{2^{n}}=h\left(X^{(n)}\right) .
$$

3.2. Relative embedding theorems. Our next objective is the following result:

Proposition 1. Let $T$ be a free ergodic $\Gamma$-action on $(X, \mathcal{F}, \mu)$ and $H$ a finite partition of $X$. Suppose that the $(n)-$ Bernoulli process $X^{(n)}$ satisfies $h\left(X^{(n)}\right) \leq$ 
$h(T)-h(T, H)$. Then there is a partition $B$ of $X$ such that dist $(T, B)=\operatorname{dist} X^{(n)}$ and $(T, B) \perp(T, H)$.

This result is a special case of a more general embedding theorem, namely the counterpart for $\Gamma$-processes of the relative Sinai theorem of Thouvenot [13]. The theorem we want can be proved by arguments that are fully analogous to those that prove Thouvenot's theorem. This being so, it is tempting to dismiss the proof as redundant. We feel, however, that omitting it would only serve to make this work less accessible (not to mention less believable), so we will give the argument in some detail. The expert reader can (and will without our suggestion) pass quickly to the proof of our main theorem in the next section.

In order to formulate the relative Sinai theorem, we need some preliminary definitions.

For $i=1,2$, let $T_{i}$ be a $\Gamma$-action defined on a space $\left(X_{i}, \mathcal{F}_{i}, \mu_{i}\right)$. Suppose that $H_{1}$ and $H_{2}$ are partitions such that $\operatorname{dist}\left(T_{1}, H_{1}\right)=\operatorname{dist}\left(T_{2}, H_{2}\right)$.

Definition 7. An $H_{1}, H_{2}$-relative joining of $T_{1}$ and $T_{2}$ is a $\Gamma$-action $S$ on a space $(Y, \mathcal{G}, \nu)$, with factor maps $\phi_{i}:(S, Y, \mathcal{G}, \nu) \rightarrow\left(T_{i}, X_{i}, \mathcal{F}_{i}, \mu_{i}\right)(i=1,2)$ such that $\left|\phi_{1}^{-1}\left(H_{1}\right)-\phi_{2}^{-1}\left(H_{2}\right)\right|_{\nu}=0$. Here $|\cdot|_{\nu}$ denotes the partition metric with respect to $\nu$.

Suppose that $T_{1}$ and $T_{2}$ are as above, and $P_{1}$ and $P_{2}$ are partitions of $X_{1}$ and $X_{2}$, respectively, with the same index set. The following three conditions are equivalent.

1. For arbitrarily large $n$, there is a measure $\nu_{n}$ on $\left(P_{1} \times H \times P_{2}\right)^{\Gamma_{n}}$ with (the obvious) marginals equal to $\operatorname{dist}\left(T_{1}, P_{1} \vee H_{1}\right)_{\Gamma_{n}}$ and $\operatorname{dist}\left(T_{2}, P_{2} \vee H_{2}\right)_{\Gamma_{n}}$, such that

$$
\frac{1}{2^{n}} \sum_{g \in \Gamma_{n}}\left|P_{1 g}-P_{2 g}\right|_{\nu_{n}}<\varepsilon
$$

Here $P_{1 g}$ (resp. $\left.P_{2 g}\right)$ denotes the partition of $\left(P_{1} \times H \times P_{2}\right)^{\Gamma_{n}}$ by $P_{1}$ (resp. $P_{2}$ ) on the $g^{\text {th }}$ coordinate.

2. There is an $H_{1}, H_{2}$-relative joining $(S, Y, \mathcal{G}, \nu)$ of the factors $\left(T_{1},\left(P_{1} \vee H_{1}\right)_{\Gamma}\right.$, $\left.\mu_{1}\right)$ and $\left(T_{2},\left(P_{2} \vee H_{2}\right)_{\Gamma}, \mu_{2}\right)$, such that, setting $P_{i}^{\prime}=\phi_{i}^{-1}\left(P_{i}\right), i=1,2$, we have $\left|P_{1}^{\prime}-P_{2}^{\prime}\right|_{\nu}<\varepsilon$.

3. There is an $H_{1}, H_{2}$-relative joining $(S, Y, \mathcal{G}, \nu)$ of $\left(T_{1}, X_{1}, \mathcal{F}_{1}, \mu_{1}\right)$ and $\left(T_{2}, X_{2}\right.$, $\left.\mathcal{F}_{2}, \mu_{2}\right)$, such that, setting $P_{i}^{\prime}=\phi_{i}^{-1}\left(P_{i}\right), i=1,2$, we have $\left|P_{1}^{\prime}-P_{2}^{\prime}\right|_{\nu}<\varepsilon$.

If the actions $T_{1}$ and $T_{2}$ are ergodic, these conditions are equivalent to:

4. There is an ergodic $H_{1}, H_{2}$-relative joining $(S,(Y, \mathcal{G}, \nu))$ of $T_{1}$ and $T_{2}$ such that $\left|P_{1}^{\prime}-P_{2}^{\prime}\right|_{\nu}<\varepsilon$.

To see that these conditions are equivalent, note that each $\nu_{n}$ in the first condition can be replaced by a $\Gamma_{n}$-invariant measure $\nu_{n}^{\prime}$ on $\left(P_{1} \times H \times P_{2}\right)^{\Gamma_{n}}$ by averaging the translates of $\nu_{n}$ by the elements of $\Gamma_{n}$. This measure $\nu_{n}^{\prime}$ still has the same marginals on $\left(P_{1} \times H\right)^{\Gamma_{n}}$ and $\left(H \times P_{2}\right)^{\Gamma_{n}}$, since they were $\Gamma_{n}$-invariant to begin with, and also has the property that, for all $g \in \Gamma_{n},\left|P_{1 g}-P_{2 g}\right|_{\nu_{n}^{\prime}}<\varepsilon$. Now $\nu_{n}^{\prime}$ immediately extends to a $\Gamma$-invariant measure $\nu_{n}^{\prime \prime}$ on $Y=(P \times H \times Q)^{\Gamma}$ by forming the independent product along cosets of $\Gamma_{n}$ in $\Gamma$, and any subsequent limit of the $\nu_{n}^{\prime \prime}$ in the weak topology will give a measure satisfying the second condition, where $S$ is translation by $\Gamma$ on $Y$. A joining of the second type can be extended to a joining of the third type by forming relatively independent products. If the 
actions $T_{1}$ and $T_{2}$ are ergodic, the fourth condition follows from the third by an application of an ergodic decomposition theorem. See for example [5] or [11].

We refer to a measure as in the first condition as a $\bar{d}_{H_{1}, H_{2}}^{2^{n}}$-match between $\operatorname{dist}\left(T_{1}, P_{1} \vee H_{1}\right)_{\Gamma_{n}}$ and $\operatorname{dist}\left(T_{2}, P_{2} \vee H_{2}\right)_{\Gamma_{n}}$ to within $\varepsilon$.

Definition 8. If the above conditions are satisfied, we write

$$
\bar{d}_{H_{1}, H_{2}}\left[\left(T_{1}, P_{1} \vee H_{1}\right),\left(T_{2}, P_{2} \vee H_{2}\right)\right]<\varepsilon .
$$

Definition 9. A $\Gamma$-process $(T, P \vee H)$ is $H$-relatively finitely determined if for all $\varepsilon>0$, there exist $\delta>0$ and $n \in \mathbb{N}$ such that for all ergodic processes $\left(S, Q \vee H^{\prime}\right)$ satisfying

1. $\# P=\# Q$,

2. $\operatorname{dist}(T, H)=\operatorname{dist}\left(S, H^{\prime}\right)$,

3. $h\left(S, Q \vee H^{\prime}\right)>h(T, P \vee H)-\delta$,

4. $\left|\operatorname{dist}(T, P \vee H)_{\Gamma_{n}}-\operatorname{dist}\left(S, Q \vee H^{\prime}\right)_{\Gamma_{n}}\right|<\delta$.

We get $\bar{d}_{H, H^{\prime}}\left[(T, P \vee H),\left(S, Q \vee H^{\prime}\right)\right]<\varepsilon$.

We show now that every $\Gamma$-process $(T, B \vee H)$ such that $\operatorname{dist}(T, B)=\operatorname{dist}\left(X^{(n)}\right)$ and $(T, B) \perp(T, H)$ is $H$-relatively finitely determined. We give this argument in some detail, since there is a slight difference between the argument here and the corresponding argument in the case of $\mathbb{Z}$-processes.

Lemma 9. For all $\varepsilon>0$, there exists $\delta>0$ such that for all finite partitions $P$ and $Q, H(P \mid Q)-H(Q)>H(P)-\delta$ implies $P \stackrel{\varepsilon}{\perp} Q$.

See [10].

Lemma 10. For all $\varepsilon>0$, there exists $\delta>0$ such that for all $\Gamma$-actions $S$, and all (finite) partitions $P$ and $H$, if

$$
h\left(S, P \vee H^{\prime}\right)>H(P)+H\left(S, H^{\prime}\right)-\delta,
$$

then for all sufficiently large $n \in \mathbb{N}$, the partitions $\left\{S^{g}\left(P \vee H^{\prime}\right)\right\}_{g \in \Gamma_{n}}$, when ordered arbitrarily as $\left\{P_{i} \vee H_{i}\right\}_{i=1}^{2^{n}}$, form a sequence such that for all but an $\varepsilon$ fraction of the indices $i$ between 1 and $2^{n}$,

$$
P_{i} \stackrel{\varepsilon}{\perp}\left(\bigvee_{j=1}^{i-1} P_{j}\right) \vee\left(\bigvee_{j=1}^{2^{n}} H_{j}\right)
$$

Proof. Fix $\varepsilon$ and choose $\delta$ as in Lemma 9 , and choose $\delta_{1}$ so that $\delta_{1}<\min \left\{\varepsilon^{2}, \delta^{2}\right\}$. Suppose $h\left(S, P \vee H^{\prime}\right)>H(P)+H\left(S, H^{\prime}\right)-\delta_{1}$. Then for all sufficiently large $n$,

$$
\frac{1}{2^{n}} H\left(\bigvee_{g \in \Gamma_{n}} S^{g}\left(P \vee H^{\prime}\right)\right)>H(P)+\frac{1}{2^{n}} H\left(\bigvee_{g \in \Gamma_{n}} S^{g} H^{\prime}\right)-\delta_{1},
$$

and therefore, writing $\Gamma_{n}=\left\{g_{i}\right\}_{i=1}^{2^{n}}$, we have 


$$
\begin{gathered}
\frac{1}{2^{n}} H\left(\bigvee_{i=1}^{2^{n}} S^{g_{i}} H^{\prime}\right)+\frac{1}{2^{n}} \sum_{k=1}^{2^{n}} H\left(S^{g_{k}} P \mid\left(\bigvee_{i=1}^{k-1} S^{g_{i}} P\right) \vee\left(\bigvee_{i=1}^{2^{n}} S^{g_{i}} H^{\prime}\right)\right) \\
>H(P)+\frac{1}{2^{n}} H\left(\bigvee_{g \in \Gamma_{n}} S^{g} H^{\prime}\right)-\delta_{1},
\end{gathered}
$$

so that

$$
\frac{1}{2^{n}} \sum_{k=1}^{2^{n}} H\left(S^{g_{k}} P \mid\left(\bigvee_{i=1}^{k-1} S^{g_{i}} P\right) \vee\left(\bigvee_{i=1}^{2^{n}} S^{g_{i}} H^{\prime}\right)\right)>H(P)-\delta_{1}
$$

Since each $H\left(S^{g_{k}} P \mid\left(\bigvee_{i=1}^{k-1} S^{g_{i}} P\right) \vee\left(\bigvee_{i=1}^{2^{n}} S^{g_{i}} H^{\prime}\right)\right) \leq H(P)$, it follows that at most a $\sqrt{\delta_{1}}-$ fraction of the indices $k$ could give

$$
H\left(S^{g_{k}} P \mid\left(\bigvee_{i=1}^{k-1} S^{g_{i}} P\right) \vee\left(\bigvee_{i=1}^{2^{n}} S^{g_{i}} H^{\prime}\right)\right) \leq H(P)-\sqrt{\delta_{1}}
$$

Since $\sqrt{\delta_{1}}<\min \{\varepsilon, \delta\}$, the previous lemma completes the proof.

Lemma 11. Let $(T, B \vee H)$ be a $\Gamma$-process such that $(T, B)$ is a process with $\operatorname{dist}(T, B)=\operatorname{dist} X^{(n)}$, which is also independent of $(T, H)$. Then $(T, B \vee H)$ is $H$-relatively finitely determined.

Proof. Fix $\varepsilon>0$. Choose $\delta^{\prime}$ subject to conditions to follow. Suppose $\left(S, P \vee H^{\prime}\right)$ is a $\Gamma$-process satisfying

1. for all $n$, dist $(T, H)_{\Gamma_{n}}=\operatorname{dist}\left(S, H^{\prime}\right)_{\Gamma_{n}}$,

2. $\left|\operatorname{dist}(T, B)_{\Gamma_{n}}-\operatorname{dist}(S, P)_{\Gamma_{n}}\right|<\delta^{\prime}$,

3. $h\left(S, P \vee H^{\prime}\right)>h(T, B \vee H)^{\prime}-\delta^{\prime}$.

We wish to show that

$$
\bar{d}_{H, H^{\prime}}\left((T, B \vee H),\left(S, P \vee H^{\prime}\right)\right)<\varepsilon .
$$

For notational convenience, let $P_{n}=(S, P)_{\Gamma_{n}}, H_{n}^{\prime}=\left(S, H^{\prime}\right)_{\Gamma_{n}}, B_{n}=(T, B)_{\Gamma_{n}}$ and $H_{n}=(T, H)_{\Gamma_{n}}$. If $\delta^{\prime}$ was chosen sufficiently small, then $h\left(S^{(n)}, P_{n} \vee H_{n}^{\prime}\right)>$ $H\left(P_{n}\right)+H\left(S^{(n)}, H_{n}^{\prime}\right)-\delta$, where $\delta$ is the quantity in Lemma 10. According to Lemma 10, for all sufficiently large $m>n$, the partitions $\left\{S^{(n) g}\left(P_{n} \vee H_{n}^{\prime}\right)\right\}_{g \in \Gamma_{m-n}}$, however ordered, form a nearly $\varepsilon$-independent sequence (in the sense made precise in Lemma 10). This condition, together with the condition that $\left|\operatorname{dist} B_{n}-\operatorname{dist} P_{n}\right|<$ $\delta^{\prime}$, allow us to exhibit a $\bar{d}_{H, H^{\prime}}^{2^{m}}$-match showing that $\bar{d}_{H, H^{\prime}}\left((T, B \vee H),\left(S, P \vee H^{\prime}\right)\right)$ $<\varepsilon$.

We do this in the usual way, inductively adding partitions to a space to exhibit the match. Briefly, having ordered $\Gamma_{m-n}$ arbitrarily as $\left\{g_{i}\right\}_{i=1}^{2^{m-n}}$, we fix a probability space $(Z, \nu)$. Having built partitions $\left\{B_{n, i}^{\prime \prime}\right\}_{i=1}^{k},\left\{P_{n, i}^{\prime \prime}\right\}_{i=1}^{k}$, and $\left\{H_{n, i}^{\prime \prime}\right\}_{i=1}^{2^{m-n n}}$, to match the distributions $\operatorname{dist}\left(\bigvee_{i=1}^{k} T^{(n) g_{i}}\left(B_{n} \vee H_{n}\right)\right), \operatorname{dist}\left(\bigvee_{i=1}^{k} S^{(n) g_{i}}\left(P_{n} \vee H_{n}^{\prime}\right)\right)$ and dist $\left(\bigvee_{i=1}^{2^{m-n}} T^{(n) g_{i}} H_{n}\right)$, we construct partitions $P_{n, k+1}^{\prime \prime}$ and $B_{n, k+1}^{\prime \prime}$ by conditioning on each atom $a$ of $\left(\bigvee_{i=1}^{k} P_{n, i}^{\prime \prime}\right) \vee\left(\bigvee_{i=1}^{k} B_{n, i}\right) \vee\left(\bigvee_{i=1}^{2^{n}} H_{n, i}\right)$, and on $a$ 
making the partition distance $\left|\left(P_{n, k+1}^{\prime \prime}\right)_{a}-\left(B_{n, k+1}^{\prime \prime}\right)_{a}\right|$ as small as the conditional distributions (given by the original processes) permit. The $\varepsilon-$ independence condition guarantees that for most indices $k$, the resulting partitions will satisfy

$$
\left|P_{n, k+1}^{\prime \prime}-B_{n, k+1}^{\prime \prime}\right|<\varepsilon \text {. }
$$

This yields a $\bar{d}_{H, H^{\prime}}^{2^{m-n}}-$ match betweeen

$$
\operatorname{dist}\left(\bigvee_{i=1}^{2^{m-n}} T^{(n) g_{i}}\left(B_{n} \vee H_{n}\right)\right) \text { and } \operatorname{dist}\left(\bigvee_{i=1}^{2^{m-n}} S^{(n) g_{i}}\left(P_{n} \vee H_{n}^{\prime}\right)\right)
$$

to within $\varepsilon$, which can simultaneously be viewed as a $\bar{d}_{H, H^{\prime}}^{m}$-match between

$$
\operatorname{dist}\left(\bigvee_{g \in \Gamma_{m}} T^{g}(B \vee H)\right) \text { and } \operatorname{dist}\left(\vee_{g \in \Gamma_{m}} S^{g}\left(P \vee H^{\prime}\right)\right)
$$

to within $\varepsilon$.

The relative Sinai theorem will follow from the following copying theorem:

Theorem 1. Let $\left(T_{i}, P_{i} \vee H_{i}\right), i=1,2$, be ergodic $\Gamma$-processes on spaces $\left(X_{i}, \mathcal{F}_{i}, \mu_{i}\right)$, such that $\operatorname{dist}\left(T_{1}, H_{1}\right)=\operatorname{dist}\left(T_{2}, H_{2}\right)$, and $h\left(T_{1}\right)=h\left(T_{2}, P_{2} \vee H_{2}\right)$. Let $T^{\prime}$ on $\left(X^{\prime}, \mathcal{F}^{\prime}, \mu^{\prime}\right)$ be an ergodic $H_{1}, H_{2}-$ joining of $T_{1}$ and $T_{2}$. For $i=1,2$, let $\phi_{i}$ denote the factor map onto $T_{i}$ and set $P_{i}^{\prime}=\phi_{i}^{-1} P_{i}$ and $H^{\prime}=\phi_{i}^{-1} H_{i}$. Suppose also that $T_{1}$ acts freely on $\left(X_{1}, \mathcal{F}_{1}, \mu_{1}\right)$. Then for all $n \in \mathbb{N}$ and $\delta>0$, there exists a partition $P_{2}^{\prime \prime} \subset \mathcal{F}_{1}$ such that

1. $\left|\operatorname{dist}\left(T_{1}, P_{1} \vee P_{2}^{\prime \prime} \vee H_{1}\right)_{\Gamma_{n}}-\operatorname{dist}\left(T^{\prime}, P_{1}^{\prime} \vee P_{2}^{\prime} \vee H^{\prime}\right)_{\Gamma_{n}}\right|<\delta$, and

2. $h\left(T_{1}, P_{2}^{\prime \prime} \vee H_{1}\right)>h\left(T_{2}, P_{2} \vee H_{2}\right)-\delta$.

If it happens that $h\left(T_{1}, P_{1} \vee H_{1}\right)=h\left(T_{2}, P_{2} \vee H_{2}\right)$, then $P_{2}^{\prime \prime}$ may be taken to be in $\left(T_{1}, P_{1} \vee H_{1}\right)_{\Gamma}$.

Proof. Fix $n \in \mathbb{N}$ and $\delta>0$. Choose a partition $R$ of $X_{1}$ so that

$$
h_{1}=h\left(T_{1}, P_{1} \vee H_{1} \vee R\right)>h\left(T_{2}, P_{2} \vee H_{2}\right)-\frac{\delta}{3}=h-\frac{\delta}{3} .
$$

The hypotheses force

$$
h\left(T_{2}, P_{2} \vee H_{2}\right) \geq h\left(T_{1}, P_{1} \vee H_{1} \vee R\right) .
$$

Choose $\eta_{1}>0$ such that $\frac{1}{2}>\eta_{1}$, and choose $\eta_{2}>0$, and then $m \in \mathbb{N}$, so that

1. there is a collection $C_{1} \subset\left(T_{1}, P_{1} \vee H_{1} \vee R\right)_{\Gamma_{m}}$, such that $\mu_{1}\left(\bigcup C_{1}\right)>1-\eta_{2}$, and for each $c_{1} \in C_{1}$,

$$
\mu_{1}\left(c_{1}\right)>2^{-2^{m}\left(h_{1}+\eta_{2}\right)},
$$

2. there is a collection $C_{2} \subset\left(T_{2}, P_{2} \vee H_{2}\right)_{\Gamma_{m}}$, such that $\mu_{2}\left(\bigcup C_{2}\right)>1-\eta_{2}$, and for each $c_{2} \in C_{2}$,

$$
\mu_{2}\left(c_{2}\right)<2^{-2^{m}\left(h-\eta_{2}\right)},
$$

3. there is a collection $C_{3} \subset\left(T^{\prime}, P_{1}^{\prime} \vee P_{2}^{\prime} \vee H^{\prime}\right)_{\Gamma_{m}}$, such that $\mu^{\prime}\left(\bigcup C_{3}\right)>1-\eta_{2}$, and for each $x \in c_{3} \in C_{3}$,

$$
\left|\operatorname{dist}_{\Gamma_{m}(x)}\left(T^{\prime}, P_{1}^{\prime} \vee P_{2}^{\prime} \vee H^{\prime}\right)_{\Gamma_{n}}-\operatorname{dist}\left(T^{\prime}, P_{1}^{\prime} \vee P_{2}^{\prime} \vee H^{\prime}\right)_{\Gamma_{n}}\right|<\eta_{2}
$$


For $i=1,2$, let $C_{i}^{\prime}$ denote the collection of atoms measurable with respect to $\left(T^{\prime}, P_{1}^{\prime} \vee P_{2}^{\prime} \vee H^{\prime}\right)_{\Gamma_{m}}$ that correspond to $C_{i}$.

If $\eta_{2}$ was chosen small enough, then there is a set $D_{1}^{\prime} \subset C_{1}^{\prime}$ such that $\mu^{\prime}\left(\bigcup D_{1}^{\prime}\right)>$ $1-\eta_{1}$, and each $d_{1}^{\prime} \in D_{1}^{\prime}$ is covered, up to an $\eta_{1}$ fraction of its own measure, by a collection $D_{2}^{\prime}\left(d_{1}^{\prime}\right) \subset C_{2}^{\prime}$ such that for all $c_{2}^{\prime} \in D_{2}^{\prime}\left(d_{1}^{\prime}\right),\left(d_{1}^{\prime} \cap c_{2}^{\prime}\right) \subset\left(\bigcup C_{3}^{\prime}\right)$.

Now, for every $d_{1}^{\prime} \in D_{1}^{\prime}$ and $c_{2}^{\prime} \in D_{2}^{\prime}\left(d_{1}^{\prime}\right)$, we have

$$
\frac{\mu^{\prime}\left(c_{2}^{\prime}\right)}{\mu^{\prime}\left(d_{1}^{\prime}\right)}<2^{-2^{m}\left(h-h_{1}-2 \eta_{2}\right)} \leq 2^{2^{m}\left(2 \eta_{2}\right)} \text {. }
$$

Let $L^{\prime}$ be a partition of $X^{\prime}$ consisting of $\left[2^{2^{m}\left(2 \eta_{2}\right)}\right]+2$ elements of equal measure, that is independent of $\left(T^{\prime}, P_{2}^{\prime} \vee H^{\prime}\right)_{\Gamma_{m}}$. Associating to each $d_{1}^{\prime} \in D_{1}^{\prime}$ the set of atoms of the form $l^{\prime} \cap c_{2}^{\prime}$, where $l^{\prime} \in L^{\prime}$ and $c_{2}^{\prime} \in D_{2}^{\prime}\left(d_{1}^{\prime}\right)$, an application of the marriage lemma yields a bijection $\phi: D_{1}^{\prime} \rightarrow L^{\prime} \vee\left(T^{\prime}, P_{2}^{\prime} \vee H^{\prime}\right)_{\Gamma_{m}}$ such that for each $d_{1}^{\prime} \in D_{1}^{\prime}, \phi\left(d_{1}^{\prime}\right) \subset \bigcup D_{2}^{\prime}\left(d_{1}^{\prime}\right)$. Note that for each $d_{1}^{\prime} \in D_{1}^{\prime},\left(T^{\prime}, H^{\prime}\right)_{\Gamma_{m}}\left(d_{1}^{\prime}\right)=$ $\left(T^{\prime}, H^{\prime}\right)_{\Gamma_{m}}\left(\phi\left(d_{1}^{\prime}\right)\right)$.

Let $B \subset X_{1}$ be a base of a $\Gamma_{m}$-Rokhlin tower for $T_{1}$, such that

$$
B \perp\left(T_{1}, P_{1} \vee H_{1} \vee R\right)_{\Gamma_{m}} .
$$

Let $D_{1}$ denote the collection of sets in $X_{1}$ corresponding to the collection $D_{1}^{\prime}$ in $X^{\prime}$. Similarly, if $d_{1}^{\prime} \in D_{1}^{\prime}$, we denote the corresponding subset of $X_{1}$ by $d_{1}$.

We construct the desired partition $P_{2}^{\prime \prime}$ and two auxilliary partitions $L$ and $Q_{B}$ as follows. For each $d_{1} \in D_{1}$, and each $x \in B \cap d_{1}$,

1. We set $\left(T_{1}, P_{2}^{\prime \prime}\right)_{\Gamma_{m}}(x)=\left(T^{\prime}, P_{2}^{\prime}\right)_{\Gamma_{m}}\left(\phi\left(d_{1}^{\prime}\right)\right)$, and we put all those points not in $\Gamma_{m}\left(B \cap\left(\bigcup D_{1}\right)\right)$ into a single atom of $P_{2}^{\prime \prime}$.

2. We set $L(x)=L^{\prime}\left(\phi\left(d_{1}^{\prime}\right)\right)$, and we put all those points not in $B \cap\left(\bigcup D_{1}\right)$ into a single atom of $L$.

3. We set $Q_{B}=\chi_{B}$.

It is clear that $\left(T_{1}, P_{2}^{\prime \prime} \vee H_{1} \vee L \vee Q_{B}\right)_{\Gamma_{m}} \stackrel{\eta_{1}}{\supset}\left(T_{1}, P_{1} \vee H_{1} \vee R\right)_{\Gamma_{m}}$, so that, if $\eta_{1}$ was chosen small enough, $h\left(T_{1}, P_{2}^{\prime \prime} \vee H_{1} \vee L \vee Q_{B}\right)>h\left(T_{1}, P_{1} \vee H_{1} \vee R\right)-\frac{\delta}{3}$. However, if $m$ was chosen to be sufficiently large, $H(L)+H(B)<\frac{\delta}{3}$, so that $h\left(T_{1}, P_{2}^{\prime \prime} \vee H_{1}\right)>h\left(T_{1}, P_{1} \vee H_{1} \vee R\right)-\frac{2 \delta}{3}>h\left(T_{2}, P_{2} \vee H_{2}\right)-\delta$, as desired.

Since, for each $d_{1} \in D_{1},\left(T_{1}, P_{1} \vee H_{1} \vee P_{2}^{\prime \prime}\right)_{\Gamma_{m}}$ is the name of an atom of $C_{3}$, we see that if $\eta_{1}$ and $\eta_{2}$ were chosen sufficiently small, we get

$$
\left|\operatorname{dist}\left(T_{1}, P_{1} \vee P_{2}^{\prime \prime} \vee H_{1}\right)_{\Gamma_{n}}-\operatorname{dist}\left(T^{\prime}, P_{1}^{\prime} \vee P_{2}^{\prime} \vee H^{\prime}\right)_{\Gamma_{n}}\right|<\delta .
$$

If it had been the case that $h\left(T_{1}, P_{1} \vee H_{1}\right)=h\left(T_{2}, P_{2} \vee H_{2}\right)$, then $R$ could have been taken to be the trivial partition, and the Rokhlin tower could have been chosen to be measurable with respect to $\left(T_{1}, P_{1} \vee H_{1}\right)_{\Gamma}$, so that we would get $P_{2}^{\prime \prime} \subset\left(T_{1}, P_{1} \vee H_{1}\right)_{\Gamma}$.

The relative Sinai theorem can now be stated as follows:

Theorem 2. Suppose that $(T, P \vee H)$ is an ergodic $H$-relatively finitely determined $\Gamma$-process on a space $(X, \mathcal{F}, \mu)$ and $S$ is a free ergodic action on a space $(Y, \mathcal{G}, \nu)$ and $H^{\prime}$ a partition of $Y$ such that $\operatorname{dist}(T, H)=\operatorname{dist}\left(S, H^{\prime}\right)$ and $h(S) \geq$ $h(T, P \vee H)$. Then there is a partition $P^{\prime}$ of $Y$ such that $\operatorname{dist}\left(S, P^{\prime} \vee H^{\prime}\right)=$ $\operatorname{dist}(T, P \vee H)$. 
Since, as we showed in Lemma 11, the direct product of the $(n)$-Bernoulli process $X^{(n)}$ with an arbitrary ergodic $\Gamma$-process $(T, H)$ is $H$-relatively finitely determined, proposition 1 will now follow.

Lemma 12. Suppose that for $i=1,2, T_{i}$ is an ergodic $\Gamma$-action on the space $\left(X_{i}, \mathcal{F}_{i}, \mu_{i}\right)$ and $H_{i}$ is a partition such that dist $\left(T_{1}, H_{1}\right)=\operatorname{dist}\left(T_{2}, H_{2}\right)$. Then there exists an ergodic $H$-relative joining of $T_{1}$ and $T_{2}$.

Proof. The arguments showing the equivalence of the four relative $\bar{d}$ conditions prove this lemma as well.

Proof of Theorem 2. Since we can choose a partition $Q$ of $Y$ with $h\left(S, Q \vee H^{\prime}\right)=$ $h(T, P \vee H)$, and replace $(Y, \mathcal{G}, \nu)$ by $\left(Y,\left(S, Q \vee H^{\prime}\right)_{\Gamma}, \nu\right)$, we may assume, without loss of generality that $h(S)=h(T, P \vee H)$. Fix a sequence of positive numbers $\varepsilon_{i}$ such that $\sum_{i} \varepsilon_{i}<\infty$. Fix an ergodic $H, H^{\prime}-$ relative joining $\lambda_{0}$ of $T$ and $S$. Since $(T, P \vee H)$ is $H$-relatively finitely determined, an application of the copying lemma will give us a partition $P_{1}$ of $Y$ such that $d_{H^{\prime}, H}\left[\left(S, P_{1} \vee H^{\prime}\right),(T, P \vee H)\right]<\varepsilon_{1}$. Therefore, this relative $\bar{d}$ condition and Lemma 12 give us an ergodic joining $\lambda_{1}$ (invariant under an action $\bar{T}$ ) of $S$ and $T$ such that (denoting the partitions in the joining's space corresponding to $H, P_{1}$ and $P$ by $\bar{H}, \overline{P_{1}}$ and $\left.\bar{P}\right)\left|\overline{P_{1}}-\bar{P}\right|_{\lambda_{1}}<\varepsilon_{1}$. A second application of the copying lemma gives us, for arbitrarily chosen $n$ and $\delta$, a partition $P_{2}$ of $Y$ such that

$$
\left|\operatorname{dist}\left(S, P_{1} \vee P_{2} \vee H^{\prime}\right)_{\Gamma_{n}}-\operatorname{dist}\left(\bar{T}, \overline{P_{1}} \vee \bar{P} \vee \bar{H}\right)_{\Gamma_{n}}\right|<\delta
$$

and

$$
h\left(S, P_{2} \vee H^{\prime}\right)>h(T, P \vee H)-\delta .
$$

In particular, we can obtain $P_{2}$ such that

$$
d_{H^{\prime}, H}\left[\left(S, P_{2} \vee H^{\prime}\right),(T, P \vee H)\right]<\varepsilon_{2}
$$

and

$$
\left|P_{1}-P_{2}\right|<\varepsilon_{1}
$$

Continuing in this fashion, we obtain a sequence of partitions $\left\{P_{i}\right\}_{i=1}^{\infty}$ of $Y$ such that

$$
d_{H^{\prime}, H}\left[\left(S, P_{i} \vee H^{\prime}\right),(T, P \vee H)\right]<\varepsilon_{i}
$$

and

$$
\left|P_{i}-P_{i+1}\right|<\varepsilon_{i} \text {. }
$$

Therefore we can set $P^{\prime}=\lim _{i \rightarrow \infty} P_{i}$ and we get

$$
\operatorname{dist}\left(S, P^{\prime} \vee H^{\prime}\right)=\operatorname{dist}(T, P \vee H) \text {. }
$$

Although we will not need it in the proof of our main theorem, we include here a proof of the relative isomorphism theorem for $\Gamma$-actions. First we note a strengthening of the relative Sinai theorem. 
Theorem 3. Suppose that $(T, P \vee H)$ is an ergodic $H$-relatively finitely determined $\Gamma$-process on a space $(X, \mathcal{F}, \mu)$ and $S$ is a free ergodic action on a space $(Y, \mathcal{G}, \nu)$ and $H^{\prime}$ a partition of $Y$ such that dist $(T, H)=\operatorname{dist}\left(S, H^{\prime}\right)$ and $h(S) \geq$ $h(T, P \vee H)$. Let $Q$ be another partition of $Y$ and $\lambda$ an $H, H^{\prime}$-relative ergodic joining of $S$ and $T$, invariant under an action $\bar{T}$. Then for all $\varepsilon>0$ and $n \in \mathbb{N}$ there is a partition $P^{\prime}$ of $Y$ such that dist $\left(S, P^{\prime} \vee H^{\prime}\right)=\operatorname{dist}(T, P \vee H)$ and

$$
\left|\operatorname{dist}\left(S, P^{\prime} \vee Q \vee H^{\prime}\right)_{\Gamma_{n}}-\operatorname{dist}(\bar{T}, \bar{P} \vee \bar{Q} \vee \bar{H})_{\Gamma_{n}}\right|<\varepsilon \text {. }
$$

(As before we let $\bar{P}, \bar{Q}$, and $\bar{H}$ denote the partitions in the joining corresponding to $P, Q$, and $H$.)

Proof. Fix $\varepsilon>0$ and $n \in \mathbb{N}$. In the proof of the relative Sinai theorem, at the first application of the copying theorem, we are free to choose the first approximating partition $P_{1}$ to make

$$
\left|\operatorname{dist}\left(S, P_{1} \vee Q \vee H^{\prime}\right)_{\Gamma_{n}}-\operatorname{dist}(\bar{T}, \bar{P} \vee \bar{Q} \vee \bar{H})_{\Gamma_{n}}\right|<\frac{\varepsilon}{2},
$$

and to make

$$
\bar{d}_{H^{\prime}, H}\left[\left(S, P_{1} \vee H^{\prime}\right),(T, P \vee H)\right]<\frac{\varepsilon}{2^{n+1}} .
$$

In the $i^{\text {th }}$ application of the copying theorem, $i>1$, we can choose $P_{i}$ so that

$$
\left|P_{i}-P_{i-1}\right|<\frac{\varepsilon}{2^{n+i}}
$$

and

$$
\bar{d}_{H^{\prime}, H}\left[\left(S, P_{i} \vee H^{\prime}\right),(T, P \vee H)\right]<\frac{\varepsilon}{2^{n+i+1}} .
$$

As a result, the limiting partition $P^{\prime}$ satisfies $\left|P^{\prime}-P\right|<\frac{\varepsilon}{2^{n}}$, and therefore

$$
\left|\operatorname{dist}\left(S, P^{\prime} \vee Q \vee H^{\prime}\right)_{\Gamma_{n}}-\operatorname{dist}(\bar{T}, \bar{P} \vee \bar{Q} \vee \bar{H})_{\Gamma_{n}}\right|<\varepsilon \text {. }
$$

Theorem 4. Suppose that $(T, P \vee H)$ is an ergodic $H$-relatively finitely determined $\Gamma$-process on a space $(X, \mathcal{F}, \mu)$ and $\left(S, Q \vee H^{\prime}\right)$ is an ergodic $H^{\prime}$-relatively finitely determined $\Gamma$-process on a space $(Y, \mathcal{G}, \nu)$ with $\operatorname{dist}(T, H)=\operatorname{dist}\left(S, H^{\prime}\right)$ and $h\left(S, Q \vee H^{\prime}\right)=h(T, P \vee H)$. Assume further that $\mathcal{F}=(T, P \vee H)_{\Gamma}$ and $\mathcal{G}=\left(S, Q \vee H^{\prime}\right)_{\Gamma}$, and that both $T$ and $S$ act freely on the $\sigma$-algebras $\mathcal{F}$ and $\mathcal{G}$. Then $(T, P \vee H)$ and $\left(S, Q \vee H^{\prime}\right)$ are $H, H^{\prime}$-relatively isomorphic. That is, there is an isomorphism $\phi$ from $T$ to $S$ such that $\phi(H)=H^{\prime}$.

Proof. It is sufficient for us to produce a partition $P^{\prime}$ of $Y$ such that

$$
\operatorname{dist}\left(S, P^{\prime} \vee H^{\prime}\right)=\operatorname{dist}(T, P \vee H) \text {, }
$$

and

$$
Q \subset\left(S, P^{\prime} \vee H^{\prime}\right)_{\Gamma}
$$

By the relative Sinai theorem, we can choose a partition $P_{1}$ of $Y$ so that

$$
\operatorname{dist}\left(S, P_{1} \vee H^{\prime}\right)=\operatorname{dist}(T, P \vee H) \text {. }
$$

Fix a sequence of positive numbers $\left\{\varepsilon_{i}\right\}_{i=1}^{\infty}$ such that $\sum_{i} \varepsilon_{i}<\infty$. Choose a finite code $\pi_{1}$ so that

$$
P_{1} \stackrel{\pi_{1}, \varepsilon_{1}}{\complement}\left(S, Q \vee H^{\prime}\right)_{\Gamma}
$$


The action $(S, \nu)$ with partitions $Q, P_{1}$ and $H^{\prime}$ provides an ergodic $H, H^{\prime}$-relative joining of $(T, P \vee H)$ and $\left(S, Q \vee H^{\prime}\right)$. An application of the previous theorem then provides a partition $Q_{1} \subset(T, P \vee H)_{\Gamma}$ such that

$$
\operatorname{dist}\left(S, Q \vee H^{\prime}\right)=\operatorname{dist}\left(T, Q_{1} \vee H\right)
$$

and

$$
P \stackrel{\pi_{1}, \varepsilon_{1}}{\subset}\left(T, Q_{1} \vee H\right)_{\Gamma}
$$

Now choose a finite code $\rho_{1}$ so that

$$
Q_{1} \stackrel{\rho_{1}, \varepsilon_{1}}{\subset}(T, P \vee H)_{\Gamma}
$$

In the same manner, by repeated applications of the previous theorem we obtain partitions $\left\{P_{i}\right\}_{i=1}^{\infty}$ of $Y$ and $\left\{Q_{i}\right\}_{i=1}^{\infty}$ of $X$ and finite codes $\left\{\pi_{i}\right\}_{i=1}^{\infty}$ and $\left\{\rho_{i}\right\}_{i=1}^{\infty}$ such that for each $i$,

$$
\operatorname{dist}\left(S, P_{i} \vee H^{\prime}\right)=\operatorname{dist}(T, P \vee H),
$$

and for each $j \leq i+1$,

$$
P_{j} \stackrel{\pi_{j}, \varepsilon_{j}}{\complement}\left(S, Q \vee H^{\prime}\right)_{\Gamma}
$$

and for all $j \leq i$,

$$
Q \stackrel{\rho_{j}, \varepsilon_{j}}{\subset}\left(S, P_{i+1} \vee H^{\prime}\right)_{\Gamma},
$$

and (nearly) symmetrically,

$$
\operatorname{dist}\left(T, Q_{i} \vee H\right)=\operatorname{dist}\left(S, Q \vee H^{\prime}\right),
$$

and for all $j \leq i+1$,

$$
Q_{i+1} \stackrel{\rho_{j}, \varepsilon_{j}}{\complement}(T, P \vee H)_{\Gamma}
$$

and for all $j \leq i+1$,

$$
P \stackrel{\pi_{j}, \varepsilon_{j}}{\complement}\left(T, Q_{i+1} \vee H\right)_{\Gamma}
$$

Thus, for each $i$,

$$
P_{i+1} \stackrel{\pi_{i}, \varepsilon_{i}}{\complement}\left(S, Q \vee H^{\prime}\right)_{\Gamma} \text { and } P_{i} \stackrel{\pi_{i}, \varepsilon_{i}}{\complement}\left(S, Q \vee H^{\prime}\right)_{\Gamma},
$$

so that

$$
\left|P_{i}-P_{i+1}\right|<2 \varepsilon_{i} .
$$

Therefore, the partitions $P_{i}$ converge to a partition $P^{\prime}$, which must satisfy

$$
\operatorname{dist}\left(S, P^{\prime} \vee H^{\prime}\right)=\operatorname{dist}(T, P \vee H) \text {. }
$$

However, we also have that, for every $j$,

$$
Q \stackrel{\rho_{j}, \varepsilon_{j}}{\complement}\left(S, P^{\prime} \vee H^{\prime}\right)_{\Gamma}
$$

Consequently, $Q \subset\left(S, P^{\prime} \vee H^{\prime}\right)_{\Gamma}$, and we are done. 
Note that the hypotheses of Theorem 4 only require that the actions be free on the full $\sigma$-algebras. Therefore, we still may obtain relative isomorphisms even if the processes $(T, P)$ and $(S, Q)$ in the statement of the theorem are not themselves free. For example, if we suppose that the processes $(T, P \vee H)$ and $\left(S, P^{\prime} \vee H^{\prime}\right)$ in the statement of the theorem satisfy (in addition to the conditions stated there)

$$
\text { for some } n, \quad \operatorname{dist}(T, P)=\operatorname{dist} X^{(n)}
$$

and

$$
(T, P)_{\Gamma} \perp(T, H)_{\Gamma}
$$

and

$$
T \text { acts freely on }(T, H)_{\Gamma} \text {, }
$$

while

$$
(S, Q) \text { is an independent process }
$$

and

$$
(S, Q)_{\Gamma} \perp\left(S, H^{\prime}\right)_{\Gamma}
$$

and

$$
h(S, Q)=h(T, P),
$$

then $(T, P \vee H)$ and $\left(S, Q \vee H^{\prime}\right)$ are $H, H^{\prime}$-relatively isomorphic, even though the finitely determined processes $(T, P)$ and $(S, Q)$ are not isomorphic.

Some comments about the arguments leading to Theorems 2 and 4 may be in order. As in the case of $\mathbb{Z}$-actions, we do not need to use joinings of $\Gamma$-processes, nor theorems we applied to them such as the ergodic decomposition theorem, but could have kept the coding arguments on the level of finite distributions, as in the first description of the relative $\bar{d}$ distance. We made use of these technical devices because they seem to us to simplify and clarify the proof. We have not, however, chosen to give the formulation of the proof that makes use of the Baire property on an appropriate space of joinings, since we felt this would not, at least in our hands, improve the exposition.

\section{Proof of the main theorem.}

Theorem 5. Let $T$ be a free ergodic action of $\Gamma$ on $(X, \mathcal{F}, \mu)$ such that $h(T)>0$. Then there is an action $S$ of $\Gamma$, dyadically equivalent to $T$, with completely positive entropy.

Before giving the proof of this theorem, we make a change in our point of view, and introduce the corresponding terminology.

It is clear that two actions $T$ and $S$ of $\Gamma$ are dyadically equivalent if and only if they can be realized on the same space $(X, \mathcal{F}, \mu)$ so that, for almost every $x$, and every $n \in \mathbb{N}, T^{\Gamma_{n}}(x)=S^{\Gamma_{n}}(x)$. When $T$ and $S$ are so realized, $S$ is determined by $T$ and the function $\alpha: X \times \Gamma \rightarrow \Gamma$ which satisfies,

$$
T^{g}(x)=S^{\alpha(x, g)}(x),
$$

for almost all $x \in X$ and all $g \in \Gamma$. In this case, $\alpha$ must satisfy, in addition,

1. $\alpha$ is measurable in $x$,

2. for almost all $x \in X$, and for all $n \in \mathbb{N}, \alpha(x, \cdot): \Gamma_{n} \rightarrow \Gamma_{n}$ bijectively, and

3. $\alpha(x, g) \alpha\left(T^{g} x, k\right)=\alpha(x, g k)$, for almost all $x \in X$ and all $g, k \in \Gamma$. 
Conversely, given $T$ and a function $\alpha: X \times \Gamma \rightarrow \Gamma$ satisfying these three properties, we obtain an action $T_{\alpha}$, given by the above equation, which is dyadically equivalent to $T$. The function $\alpha$ is referred to as the dyadic cocycle relating $T$ and $T_{\alpha}$. Thus, abstractly given actions $T$ and $S$ are dyadically equivalent if and only if there is a dyadic cocycle $\alpha$ for $T$ such that $T_{\alpha}$ is isomorphic to $S$. In order to prove the theorem, we will construct a dyadic cocyle $\alpha$ for $T$ such that $T_{\alpha}$ has completely positive entropy.

In the event that $T$ and $S$ are related by a dyadic cocycle $\alpha$, and there is a measurable function $f: X \rightarrow \Gamma$ such that, for almost all $x$ and all $g$,

$$
\alpha(x, g)=f(x) g f\left(T^{g} x\right),
$$

we refer to $\alpha$ as a coboundary and say $T$ and $S$ are cohomologous. (Since every element of $\Gamma$ has order 2, the usual exponent of -1 may be omitted from this equation.) In this case, the map $x \mapsto T^{f(x)}(x)$ is an isomorphism from $T$ to $S$.

If a sequence of dyadic cocycles $\left\{\alpha_{k}\right\}_{k=1}^{\infty}$ for an action $T$ of $\Gamma$ converges pointwise almost everywhere, for each $g \in \Gamma$, then the function $\alpha(x, g)=\lim _{k \rightarrow \infty} \alpha_{k}(x, g)$ is again a dyadic cocycle for $T$. One can show that every dyadic cocycle can be obtained as a pointwise limit of dyadic coboundaries, and in the proof of the theorem, the desired cocyle will be obtained in this way.

Proof of the theorem. Fix an increasing sequence of finite partitions $\left\{P_{i}\right\}_{i=1}^{\infty}$ of $X$, such that $\bigvee_{i=1}^{\infty} P_{i}=X$. Fix a decreasing sequence of positive numbers $\varepsilon_{i}$ converging to 0 .

Using Lemma 6, we can choose a partition $Q_{1}$ satisfying $\left|Q_{1}-P_{1}\right|<\varepsilon_{1}$ and $h\left(T, Q_{1}\right)<h(T)$. Fix $\eta_{1}>0$, whose size will be determined shortly. Choose $n_{1}$ so that for a set $A_{1}$ with $\mu\left(A_{1}\right)>1-\eta_{1}$, and all $x \in A_{1}$,

$$
\left|\operatorname{dist}_{\Gamma_{n_{1}}(x)} Q_{1}-\operatorname{dist} Q_{1}\right|<\eta_{1} \text {. }
$$

In addition, choose $n_{1}$ so that the $\left(n_{1}\right)$-Bernoulli $\Gamma$-process described in section 3 has entropy less than $h(T)-h\left(T, Q_{1}\right)$. Applying proposition 1, we may choose a partition $B_{1}$ so that $\left(T, B_{1}\right)$ is the $\left(n_{1}\right)$-Bernoulli $\Gamma$-process, and is independent of $\left(T, Q_{1}\right)$. We define a cocycle $\alpha_{1}$ by setting, for all $x \in X$ and $\gamma \in \Gamma$,

$$
\alpha_{1}(x, g)=B_{1}(x) g B_{1}\left(T^{g}(x)\right) .
$$

Let $T_{1}$ denote the action determined by $T$ and $\alpha_{1}$ (what we called $T_{\alpha_{1}}$ in the discussion above). We now verify that, if $\eta_{1}$ is sufficiently small, then for every $L>n_{1}$,

$$
Q_{1} \stackrel{\varepsilon_{1}}{\perp}\left(T_{1}, Q_{1}\right)_{\Gamma_{L} \backslash \Gamma_{n_{1}}} .
$$

Fix $L>n_{1}$. Choose a $\left(T, \Gamma_{n_{1}}\right)-$ Rokhlin tower $\tau_{1}$ of full measure whose base $D_{1}$ is independent of the partition

$$
\left(T, Q_{1} \vee B_{1}\right)_{\Gamma_{L}}
$$

Let $R$ denote the coarser partition

$$
\left(T, Q_{1} \vee B_{1}\right)_{\Gamma_{L} \backslash \Gamma_{n_{1}}} \vee\left(T, Q_{1}\right)_{\Gamma_{n_{1}}} .
$$

Fix an atom $r$ of $\left.R\right|_{D_{1}}$. The points in the column $\Gamma_{n_{1}} r$ have $\left(T_{1}, Q_{1}\right)_{\Gamma_{L} \backslash \Gamma_{n_{1}}}$-names that are $\Gamma_{n_{1}}$-rotations of one another, and the particular such name that a point $x \in \Gamma_{n_{1}} r$ acquires is determined by the value of $B_{1}(x)$, which, we note, is constant on $\Gamma_{n_{1}}(x)$. 
Fix a particular abstract $\left(Q_{1}\right)_{\Gamma_{L} \backslash \Gamma_{n_{1}}}$-name $c$ in the $\Gamma_{n_{1}}$-rotational equivalence class determined by $r$. Let

$$
G(c)=\left\{k \in \Gamma_{n_{1}} \mid k c=c\right\},
$$

a subgroup of $\Gamma_{n_{1}}$. A point $x$ in a level $T^{h} r$ of this column has $\left(T_{1}, Q_{1}\right)_{\Gamma_{L} \backslash \Gamma_{n_{1}}}(x)=c$ if and only if $B_{1}(x)$ lies in a certain coset of $G(c, h)$ in $\Gamma_{n_{1}}$. Note that the value $G(c, h)$ is constant on the level $T^{h} r$. Since

$$
\left.\left.\left(\left(T, B_{1}\right)_{\Gamma_{n_{1}}}\right)\right|_{D_{1}} \perp R\right|_{D_{1}},
$$

and each value of $B_{1}$ occurs with equal probability, we compute that a fraction $\# G(c, h) / \# \Gamma_{n_{1}}$ of this level (and similarly each level) acquires the $\left(T_{1}, Q_{1}\right)_{\Gamma_{L} \backslash \Gamma_{n_{1}}}$ name $c$. Thus, conditioning on a single column $\Gamma_{n_{1}} r$, the distribution of $Q_{1}$ on each atom of $\left(T_{1}, Q_{1}\right)_{\Gamma_{L} \backslash \Gamma_{n_{1}}}$ equals the distribution of $Q_{1}$ on the column itself. In other words, for each atom $\left.r \in R\right|_{D_{1}}$ and each $c \in\left(T_{1}, Q_{1}\right)_{\Gamma_{L} \backslash \Gamma_{n_{1}}}$, where $c$ is in the $\Gamma_{n_{1}}$-rotational class determined by $r$,

$$
\operatorname{dist}_{\Gamma_{n_{1}} r \cap c} Q_{1}=\operatorname{dist}_{\Gamma_{n_{1}} r} Q_{1}
$$

By the choice of $n_{1}$, there is a set $\mathcal{S}$ of atoms of $\left.R\right|_{D_{1}}$ with $\mu(\bigcup \mathcal{S})>\left(1-\eta_{1}\right) \mu\left(D_{1}\right)$, such that for all $r \in \mathcal{S}$,

$$
\mid \text { dist }_{\Gamma_{n_{1}}{ }_{r} Q_{1}-\operatorname{dist} Q_{1} \mid<\eta_{1}} .
$$

Therefore, if $\eta_{1}$ is chosen sufficiently small, there is a set of atoms of $\left(T_{1}, Q_{1}\right)_{\Gamma_{L} \backslash \Gamma_{n_{1}}}$ whose union has measure greater than $1-\varepsilon_{1}$ and each atom of which has conditional distribution of $Q_{1}$ within $\varepsilon_{1}$ of dist $Q_{1}$.

We note that $h\left(T_{1}, Q_{1} \vee B_{1}\right)<h(T)$. Indeed, if we let $\varphi$ denote the isomorphism from $T_{1}$ to $T$ given by $\varphi(x)=T^{B_{1}(x)}(x)$, and let $Q_{1}^{\prime}=\varphi\left(Q_{1}\right)$, then, since $B_{1}=$ $\varphi\left(B_{1}\right)$ and $\left(T, Q_{1} \vee B_{1}\right)_{\Gamma_{n_{1}}} \supset Q_{1}^{\prime}$, we have

$$
h\left(T_{1}, Q_{1} \vee B_{1}\right)=h\left(T, Q_{1}^{\prime} \vee B_{1}\right) \leq h\left(T, Q_{1} \vee B_{1}\right)<h(T) .
$$

The construction continues as follows. After $i$ steps, we have actions $T=$ $T_{0}, T_{1}, \ldots, T_{i}$, and dyadic coboundaries $\alpha_{i}$ relating $T_{0}$ to $T_{i}$. (That is, $T_{i}^{\alpha_{i}(x, g)}(x)=$ $T_{0}^{g}(x)$ a.e. $)$ We also have integers $n_{1}<n_{2}<\ldots<n_{k}<\ldots<n_{i}$ and partitions $Q_{k}$ and $B_{k}, k=1,2, \ldots, i$, such that for $k=1,2, \ldots, i$

1. $Q_{k} \stackrel{\varepsilon_{k}}{\supset} P_{k}$ and $Q_{k} \supset Q_{k-1}$ (regarding $Q_{0}$ as the trivial partition).

2. $\left(T_{k}, B_{k}\right)$ is an $\left(n_{k}\right)$-Bernoulli process.

3. $\left(T_{k-1}, B_{k}\right) \perp\left(T_{k-1},\left(\bigvee_{l=0}^{k-1} Q_{l+1} \vee B_{l}\right)\right)=\left(T_{k-1},\left(Q_{k} \vee\left(\bigvee_{l=0}^{k-1} B_{l}\right)\right)\right)$ (regarding $B_{0}$ as the trivial partition).

4. $h\left(T_{k},\left(\bigvee_{l=1}^{k} Q_{l} \vee B_{l}\right)\right)=h\left(T_{k}, Q_{k} \vee\left(\bigvee_{l=1}^{k} B_{l}\right)\right)<h\left(T_{k}\right)=h(T)$. (For $k<$ $i$, this is implied by the previous line.)

5. $\alpha_{k}(x, g)=B_{k}(x) \alpha_{k-1}(x, g) B_{k}\left(T_{0}^{g} x\right)\left(\right.$ here $\left.\alpha_{0}(x, g) \equiv g\right)$.

6. For all $L>n_{k},\left(T_{k}, Q_{k}\right)_{\Gamma_{L} \backslash \Gamma_{n_{k}}} \perp^{\varepsilon_{k}} Q_{k}$.

Applying Lemma 6 , take a partition $\widetilde{Q}_{i+1}$ such that

$$
\left|\widetilde{Q}_{i+1}-P_{i+1}\right|<\varepsilon_{i+1}
$$


and

$$
h\left(T_{i}, \widetilde{Q}_{i+1} \vee Q_{i} \vee\left(\bigvee_{k=1}^{i} B_{k}\right)\right)<h\left(T_{i}\right)
$$

Let $Q_{i+1}=\widetilde{Q}_{i+1} \vee Q_{i}$.

We continue as in the first step of the proof. Choose $\eta_{i+1}>0$ and $n_{i+1} \in \mathbb{N}$ larger than $n_{i}$ so that for a set $A_{i+1} \subset X$ with $\mu\left(A_{i+1}\right)>1-\eta_{i+1}$, and all $x \in A_{i+1}$,

$$
\left|\operatorname{dist}_{\Gamma_{n_{i}}(x)} Q_{i+1}-\operatorname{dist} Q_{i+1}\right|<\eta_{i+1} \text {. }
$$

(Here we are referring to the action $T_{i}$, although the same condition will hold for all the previously constructed actions.) In addition, choose $n_{i+1} \in \mathbb{N}$ large enough so that by Proposition 1 we may choose a partition $B_{i+1}$ such that $\left(T_{i}, B_{i+1}\right)$ is an $\left(n_{i+1}\right)$-Bernoulli process such that

$$
\left(T_{i}, B_{i+1}\right) \perp\left(T_{i}, Q_{i+1} \vee\left(\bigvee_{l=1}^{i} B_{l}\right)\right)
$$

and

$$
h\left(T_{i}, Q_{i+1} \vee\left(\bigvee_{l=1}^{i+1} B_{l}\right)\right)<h\left(T_{k}\right) .
$$

Exactly as before, we use the process $\left(T_{i}, B_{i+1}\right)$ to construct an action $T_{i+1}$ which is cohomologous to $T_{0}$ and given by a cocyle $\alpha_{i+1}$ satisfying the condition

$$
\alpha_{i+1}(x, g)=B_{i+1}(x) \alpha_{i}(x, g) B_{i+1}\left(T_{0}^{g} x\right) .
$$

Also as before, we verify that, provided $\eta_{i+1}$ was chosen to be sufficiently small,

$$
Q_{i+1} \stackrel{\varepsilon_{i+1}}{\perp}\left(T_{i+1}, Q_{i+1}\right)_{\Gamma \backslash \Gamma_{n_{i+1}}} .
$$

We also verify that

$$
h\left(T_{i+1}, Q_{i+1} \vee\left(\bigvee_{l=1}^{i+1} B_{l}\right)\right)<h\left(T_{i+1}\right) .
$$

It is clear that each action $T_{k}$ so constructed is dyadically equivalent to $T_{0}$. Moreover, for each $l \in \mathbb{N}$, if $k$ is so large that $n_{k}>l$, then for all $\bar{k}>k$, all $g \in \Gamma_{l}$, and all $x \in X$,

$$
\alpha_{\bar{k}}(x, g)=\alpha_{k}(x, g)
$$

Thus, the cocycles $\alpha_{k}$ converge (in fact, uniformly in $x$ ) to a dyadic cocycle $\alpha$. Let $S$ denote the action corresponding to $\alpha$. We must show that $S$ has completely positive entropy. By Lemma 8, it suffices to show that for each $k$, and for all $L>n_{k}$,

$$
\left(S, Q_{k}\right)_{\Gamma_{L} \backslash \Gamma_{n_{k}}} \perp^{\bar{\varepsilon}_{k}} Q_{k},
$$

where $\lim _{k \rightarrow \infty} \bar{\varepsilon}_{k}=0$. Fix $k$ and $L>n_{k}$. It suffices to show that for all $\bar{k}>k$,

$$
\left(T_{\bar{k}}, Q_{k}\right)_{\Gamma_{L} \backslash \Gamma_{n_{k}}} \perp^{\bar{\varepsilon}_{k}} Q_{k} .
$$

This suffices, since for all sufficiently large $\bar{k}$, the partitions $\left(T_{\bar{k}}, Q_{k}\right)_{\Gamma_{L} \backslash \Gamma_{n_{k}}}$ and $\left(S, Q_{k}\right)_{\Gamma_{L} \backslash \Gamma_{n_{k}}}$ coincide. 
Now fix $\bar{k}>k$. We claim that

$$
\left(T_{k}, Q_{k}\right)_{\Gamma_{L}} \perp \bigvee_{l=k+1}^{\bar{k}}\left(T_{k}, B_{l}\right)_{\Gamma_{L}} .
$$

Indeed, this is true for $\bar{k}=k+1$ by construction, and for $\bar{k}>k+1$, we have

$$
\begin{aligned}
\left(T_{k}, B_{\bar{k}}\right)_{\Gamma_{L}} & =\left(T_{\bar{k}-1}, B_{\bar{k}}\right)_{\Gamma_{L}} \perp\left(T_{\bar{k}-1}, Q_{\bar{k}} \vee\left(\bigvee_{j=k+1}^{\bar{k}-1} B_{j}\right)\right)_{\Gamma_{L}} \\
& \supset\left(T_{k}, Q_{k} \vee\left(\bigvee_{j=k+1}^{\bar{k}-1} B_{j}\right)\right)_{\Gamma_{L}} .
\end{aligned}
$$

Thus, we can prove the claim by induction on $\bar{k}$.

We already have that

$$
\left(T_{k}, Q_{k}\right)_{\Gamma_{L} \backslash \Gamma_{n_{k}}} \perp^{\varepsilon_{k}} Q_{k} .
$$

Thus, for a collection $\mathcal{A}$ of atoms of $\left(T_{k}, Q_{k}\right)_{\Gamma_{L} \backslash \Gamma_{n_{k}}}$ with $\mu(\mathcal{A})>1-\varepsilon_{k}$, and all $a \in \mathcal{A}$,

$$
\left|\operatorname{dist}_{a} Q_{k}-\operatorname{dist}_{k}\right|<\varepsilon_{k}
$$

By the claim we just established, for each $a \in \mathcal{A}$ and each atom $b$ of $\bigvee_{l=k+1}^{\bar{k}}\left(T_{k}, B_{l}\right)_{\Gamma_{L}}$,

$$
\mid \text { dist }_{a \cap b} Q_{k}-\operatorname{dist} Q_{k} \mid<\varepsilon_{k} \text {. }
$$

But each such $a \cap b$ is entirely contained in a single atom of $\left(T_{\bar{k}}, Q_{k}\right)_{\Gamma_{L} \backslash \Gamma_{n_{k}}}$. It follows that for a collection $\mathcal{C}$ of atoms of $\left(T_{\bar{k}}, Q_{k}\right)_{\Gamma_{L} \backslash \Gamma_{n_{k}}}$ with $\mu(\mathcal{C})>1-\sqrt{\varepsilon_{k}}$, and for each $c \in \mathcal{C}, c$ is covered up to a set of measure less than $\left(\sqrt{\varepsilon_{k}}\right) \mu(c)$ by sets $a \cap b$ of the form described above. This establishes that

$$
\left(T_{\bar{k}}, Q_{k}\right)_{\Gamma_{L} \backslash \Gamma_{n_{k}}} \perp^{\bar{\varepsilon}_{k}} Q_{k},
$$

for some $\bar{\varepsilon}_{k} \rightarrow 0$.

As is the case with Kakutani equivalence, we can prove something more.

Proposition 2. The set $[T]_{d}$ of dyadic cocycles of an ergodic $\Gamma$-action $T$ can be given a complete metric topology, with respect to which it is the closure of the dyadic coboundaries.

Also,

Proposition 3. If $T$ is ergodic and of positive entropy, then

$$
\left\{\alpha \in[T]_{d}: T_{\alpha} \text { has completely positive entropy }\right\}
$$

is a residual set in $[T]_{d}$.

We could have organized the proof of Theorem 5 along these lines, using the Baire property in $[T]_{d}$ to show the existence of a cocycle giving completely positive entropy, but instead we will use the theorem (or more precisely, its proof) to prove Proposition 3. 
Proof of Proposition 2. Let $T$ be an ergodic action of $\Gamma$ on $(X, \mathcal{F}, \mu)$. We define, for each $n \in \mathbb{N}$, a pseudometric $\rho_{n}$ on $[T]_{d}$ by setting, for all cocyles $\alpha$ and $\beta$ in $[T]_{d}$,

$$
\rho_{n}(\alpha, \beta)=\mu\left\{x \in X:\left(\exists g \in \Gamma_{n}\right) \alpha(x, g) \neq \beta(x, g)\right\}
$$

We then set

$$
\rho(\alpha, \beta)=\sum_{n=1}^{\infty} \frac{\rho_{n}(\alpha, \beta)}{2^{n}} .
$$

One easily checks that this is a complete metric. We note that the cocycle $\alpha$ obtained in Theorem 5 was a limit in this metric of the coboundaries $\alpha_{i}$.

Let $\alpha$ be an arbitrary dyadic cocycle for $T$. Fix $n \in \mathbb{N}$ and choose a $\left(T, \Gamma_{n}\right)$ Rokhlin tower $\tau=\left\{T^{g} D\right\}_{g \in \Gamma_{n}}$. For each $x \in D$ and $g \in \Gamma_{n}$, set

$$
f\left(T^{g} x\right)=\alpha(x, g) g^{-1}=\alpha(x, g) g .
$$

This defines $f$ on almost all of $X$, so we can define a coboundary $\beta$ by

$$
\beta(z, g)=f(z) g f\left(T^{g} z\right),
$$

and we see that for almost every $z \in X$ and for all $g \in \Gamma_{n}$,

$$
\beta(z, g)=\alpha(z, g) \text {. }
$$

Thus $\rho(\alpha, \beta) \leq 2^{-n}$, and we have proved that the coboundaries are dense in $[T]_{d}$.

It follows that for every $\alpha \in[T]_{d},\left\{\alpha^{\prime} \in[T]_{d}: T_{\alpha^{\prime}}\right.$ is isomorphic to $\left.T_{\alpha}\right\}$ is dense in $[T]_{d}$. Indeed, fixing $\alpha \in[T]_{d}$ and $\varepsilon>0$, we can choose a coboundary $\beta$ so that $\rho(\alpha, \beta)<\varepsilon$. For an arbitrary coboundary $\beta^{\prime}$, let $\phi$ be an isomorphism from $T_{\beta}$ to $T_{\beta^{\prime}}$, and define a cocycle $\alpha^{\prime}$ by setting $\alpha^{\prime}(\phi(x), g)=\alpha(x, g)$. It follows that $T_{\alpha^{\prime}}$ is isomorphic to $T_{\alpha}$ and $\rho\left(\alpha^{\prime}, \beta^{\prime}\right)<\varepsilon$. Since the coboundaries are dense in $[T]_{d}$, the result follows.

We will make use of the following elementary combinatorial fact.

Lemma 13. For all $N \in \mathbb{N}$ and all $\zeta \in(0,1)$,

$$
\sum_{k \leq \zeta N}\left(\begin{array}{c}
N \\
k
\end{array}\right) \leq 2^{2 H\left(\frac{\zeta}{2}\right) N},
$$

where $H(x)=-x \log x-(1-x) \log (1-x)$.

Lemma 14. For each finite partition $P$, the map $\alpha \longmapsto h\left(T_{\alpha}, P\right)$ is continuous with respect to $\rho$.

Proof. Fix $\alpha \in[T]_{d}$, a finite partition $P$, and $\varepsilon>0$. We show there is a $\delta>0$ such that if $\rho(\alpha, \beta)<\delta$, then $h\left(T_{\beta}, P\right) \geq h\left(T_{\alpha}, P\right)-\varepsilon$. Since the reverse inequality follows from the fact that finite distributions are continuous in $\rho$ and $h\left(T_{\alpha}, P\right)$ is a decreasing limit of the terms $\frac{1}{2^{n}} H\left(T_{\alpha}, P\right)_{\Gamma_{n}}$, this will complete the proof.

Choose $n \in \mathbb{N}$ so that $\frac{n}{2^{n}}<\frac{\varepsilon}{8}$. Choose $\zeta$ so that $\zeta \log (\# P)<\frac{\varepsilon}{8}$ and $H\left(\frac{\zeta}{2}\right) 2^{n-1}$ $<\frac{\varepsilon}{8}$. Then choose $\delta>0$ so that $\rho(\alpha, \beta)<\delta$ implies that for all $x$ in a set $A_{\beta}$ satisfying $\mu\left(A_{\beta}\right)>1-\zeta$, and all $g \in \Gamma_{n}, T_{\alpha}^{g}(x)=T_{\beta}^{g}$. (Note that this is a $\Gamma_{n}$-invariant set.) Now fix $\beta \in[T]_{d}$ such that $\rho(\alpha, \beta)<\delta$. Suppose that $h\left(T_{\beta}, P\right)<$ $h\left(T_{\alpha}, P\right)-\varepsilon$. For brevity, write $h$ for $h\left(T_{\alpha}, P\right)$. Choose an arbitrary $\xi>0$. We 
show that for all sufficiently large $M$, we can find a collection $\mathcal{C}$ of fewer than $2^{2^{M}\left(h-\frac{\varepsilon}{2}\right)}$ atoms of $\left(T_{\alpha}, P\right)_{\Gamma_{M}}$ with $\mu(\bigcup \mathcal{C})>1-\xi$, a contradiction.

Choose $M$ so large that for all $x$ in a set $B$ of measure greater than $1-\xi / 2$,

$$
\frac{1}{2^{M}} \sum_{g \in \Gamma_{M}} \chi_{A_{\beta}}\left(T^{g}(x)\right)>1-\zeta
$$

and so that there is a collection $\mathcal{C}^{\prime}$ consisting of fewer than $2^{2^{M}(h-\varepsilon)}$ atoms of $\left(T_{\beta}, P\right)_{\Gamma_{M}}$, such that $\mu\left(\bigcup \mathcal{C}^{\prime}\right)>1-\xi / 2$. We have $\mu\left(B \cap\left(\bigcup \mathcal{C}^{\prime}\right)\right)>1-\xi$, and we now count how many atoms of $\left(T_{\alpha}, P\right)_{\Gamma_{M}}$ are needed to cover $B \cap\left(\bigcup \mathcal{C}^{\prime}\right)$.

For each $x \in B \cap\left(\bigcup \mathcal{C}^{\prime}\right)$, the name $\left(T_{\beta}, P\right)_{\Gamma_{M}}(x)$ is canonically partitioned into $2^{M-n}$ blocks corresponding to $\Gamma_{n}$-orbits. At most $\zeta 2^{M-n}$ of these correspond to points in the orbit of $x$ that are not in $A_{\beta}$. By Lemma 13 and the choice of $\zeta$, there are no more than

$$
\sum_{k \leq \zeta 2^{M-n}}\left(\begin{array}{c}
2^{M-n} \\
k
\end{array}\right)<2^{2^{M-n}\left(2 H\left(\frac{\zeta}{2}\right)\right)}<2^{2^{M}\left(\frac{\varepsilon}{8}\right)}
$$

ways of choosing such blocks. In each block that corresponds to points in $A_{\beta}$, the $\left(T_{\alpha}, P\right)$-name of $x$ must be a $\Gamma_{n}$-rotation of the $\left(T_{\beta}, P\right)$-name of $x$ in that block, and there are at most $2^{n}$ distinct such rotations. There are, of course, no more than $2^{M-n}$ blocks in which they can appear. In each of the other blocks there are at most $(\# P)^{2^{n}}$ names available, and there are at most $\zeta 2^{M-n}$ such blocks. Therefore, the number of $\left(T_{\alpha}, P\right)_{\Gamma_{M}}$-names that can arise from one such point $x$ is less than

$$
2^{2^{M}\left(\frac{\varepsilon}{8}\right)}\left((\# P)^{2^{n}}\right)^{\zeta 2^{M-n}}\left(2^{n}\right)^{2^{M-n}}=2^{2^{M}\left(\frac{\varepsilon}{8}\right)} 2^{2^{M}(\zeta \log (\# P))} 2^{2^{M}\left(\frac{n}{2^{n}}\right)}<2^{2^{M}\left(\frac{3 \varepsilon}{8}\right)} .
$$

Since there are fewer than $2^{2^{M}(h-\varepsilon)}$ atoms of $\left(T_{\beta}, P\right)_{\Gamma_{M}}$ that intersect $\left(B \cap\left(\cup \mathcal{C}^{\prime}\right)\right)$, we have the desired estimate.

Proof of Proposition 3. The proof is an adaptation of the proof in [3] of the corresponding fact for Kakutani equivalence. Fix a sequence $\mathcal{S}$ of finite partitions, $\mathcal{S}=\left\{Q_{i}\right\}_{i=1}^{\infty}$, which satisfy, for all $i, H\left(Q_{i}\right)<h(T)$, which are dense (with respect to the partition metric) in the space of all finite partitions $P$ of $X$ such that $H(P)<h(T)$, and none of which are the trivial partition. For each $i$ and $m$, let

$$
E_{i, m}=\left\{\alpha \in[T]_{d}: h\left(T_{\alpha}^{(m)}, Q_{i}\right)>\frac{H\left(Q_{i}\right)}{2}\right\} .
$$

(Recall the definition of $T^{(m)}$ from the proof of Lemma 7.) Let

$$
E^{\mathcal{S}}=\bigcap_{i=1}^{\infty} \bigcup_{m=1}^{\infty} E_{i, m}
$$

Arguing as in the proof of Lemma 7, we see that for all $\alpha \in E^{\mathcal{S}}$, and for all partitions $P$ of $X$ such that $H(P)<h(T), h\left(T_{\alpha}, P\right)>0$. It follows that $T_{\alpha}$ has completely positive entropy. Furthermore, the proof of Theorem 5 shows that for each $i, \bigcup_{m=1}^{\infty} E_{i, m}$ is dense in $[T]_{d}$. Indeed, given $\alpha \in[T]_{d}$ and $\varepsilon>0$, we have

$$
h\left(T_{\alpha}, Q_{i}\right) \leq H\left(Q_{i}\right)<h(T),
$$


and, by Theorem 2 of [2],

$$
h(T)=h\left(T_{\alpha}\right) .
$$

Therefore, arguing as in the proof of Theorem 5 allows us to construct a cocycle $\alpha_{1}$ such that, for some $m \in \mathbb{N}$ and for all $L>m$,

$$
Q_{i} \stackrel{\varepsilon_{1}}{\perp}\left(T_{\alpha_{1}}, Q_{i}\right)_{\Gamma_{L} \backslash \Gamma_{m}} .
$$

As in the proof of Theorem 5 , we are free to choose $\varepsilon_{1}$ as small as we wish, and then to choose $m$ as large as we wish. Choosing $\varepsilon_{1}$ small enough forces $h\left(T_{\alpha_{1}}^{(m)}, Q_{i}\right)>$ $\frac{H\left(Q_{i}\right)}{2}$, and choosing $m$ large enough forces $\rho\left(T_{\alpha}, T_{\alpha_{1}}\right) \leq \frac{1}{2^{m}}<\varepsilon$.

We remark that it really was necessary to invoke the proof of Theorem 5 here, and not merely its conclusion. This is different from the situation for $\mathbb{Z}$-actions, where the condition that $T_{\alpha}$ has completely positive entropy implies that for all partitions $Q, \lim _{m \rightarrow \infty} h\left(T_{\alpha}^{m}, Q\right)=H(Q)$. The existence of a single cocycle $\alpha$ such that $T_{\alpha}$ has completely positive entropy then immediately implies that there is a dense collection of such cocycles. As we remarked earlier, however, $\Gamma$-actions of completely positive entropy do not have this limiting behavior with respect to all partitions.

The proof will be complete when we show that, for each $i$ and $m, E_{i, m}$ is open, which we will do by showing that $h\left(T_{\alpha}^{(m)}, Q_{i}\right)$ is continuous in $\alpha$.

For each $k \in \mathbb{N}$, $\operatorname{dist}\left(T_{\alpha}^{(m)}, Q\right)_{\Gamma_{k}}$ varies continuously with $\alpha$, so one shows in the usual way that $h\left(T_{\alpha}^{(m)}, Q\right)$ is upper semi-continuous as a funcion of $\alpha$. To show the reverse, we write

$$
2^{m} h\left(T_{\alpha}, Q\right)=\lim _{n \rightarrow \infty} \frac{1}{2^{n}} H\left(\bigvee_{g \in \Gamma_{n+m}} T_{\alpha}^{g} Q\right)=\lim _{n \rightarrow \infty} \frac{1}{2^{n}} H\left(\bigvee_{i=1}^{2^{n+m}} T_{\alpha}^{g_{i}} Q\right),
$$

where we have chosen a particular ordering of $\Gamma_{n+m}$ (which is the restriction of an ordering of $\Gamma$ ). Namely, we say $g=\left\{\varepsilon_{j}\right\}_{j=1}^{\infty} \in \Gamma$ is less than $g^{\prime}=\left\{\varepsilon_{j}^{\prime}\right\}_{j=1}^{\infty} \in \Gamma$ if, in the last place where their coordinates differ, $\varepsilon_{j}=0$ and $\varepsilon_{j}^{\prime}=1$. We now rewrite this last expression as an average of conditional entropies. We introduce the following notation:

For each $j \in \mathbb{N}$, let $B_{j}=\Gamma_{j} \backslash \Gamma_{j-1}$. (Recall that backslash denotes set-theoretic difference.)

For each $i \in \mathbb{N}$, let $\left\{\delta_{j}\right\}_{j}$ be the binary digits of $i: i=\sum_{j} \delta_{j} 2^{j}$, and let $A_{i}=$ $\bigcup\left\{B_{j}: \delta_{j}=1\right\}$.

For each $i \in \mathbb{N}$, let $\mathcal{A}_{\alpha, i}^{m}=\left(T_{\alpha}^{(m)}, Q\right)_{A_{i}}$.

For each $n \in \mathbb{N}$, let $\mathcal{C}_{\alpha, n}^{m}=\left(T_{\alpha}^{(m)}, Q\right)_{\Gamma_{n}}$.

We claim that

$$
\lim _{n \rightarrow \infty} \frac{1}{2^{n}} H\left(\bigvee_{i=1}^{2^{n+m}} T_{\alpha}^{g_{i}} Q\right)=\lim _{n \rightarrow \infty} \frac{1}{2^{n}} \sum_{j=0}^{2^{m}} \sum_{i=1}^{2^{n}} H\left(Q \mid \mathcal{A}_{\alpha, i}^{m} \vee\left(\bigvee_{g \in A_{j}} T_{\alpha}^{(m) g} \mathcal{C}_{\alpha, n}^{m}\right)\right)
$$

(Here the algebra $\left(\bigvee_{g \in A_{0}} T_{\alpha}^{(m) g} \mathcal{C}_{\alpha, n}^{m}\right)$ is understood to mean the trivial algebra). This equation can be verified by applying the usual formula for conditional entropy 
to the expression on the left, first going through the partitions $T_{\alpha}^{(m) g} Q$, for $g \in \Gamma_{n}$, in the order inherited from $\Gamma_{n}$, and then returning to the partitions of the form $T_{\alpha}^{g_{2}} T_{\alpha}^{(m) g} Q, T_{\alpha}^{g_{3}} T_{\alpha}^{(m) g} Q, \ldots$ for $g \in \Gamma_{n}$, where $g_{2}, g_{3}, \ldots$ are the elements of $\Gamma_{m}$ listed according to the same order. After shifting the resulting summands by appropriate transformations $T_{\alpha}^{g}, g \in \Gamma_{n+m}$, we can put the expression in the above form.

For each $j=0,1, \ldots, 2^{m}-1$, the sequence

$$
\frac{1}{2^{n}} \sum_{i=1}^{2^{n}} H\left(Q \mid \mathcal{A}_{\alpha, i}^{m} \vee\left(\bigvee_{g \in A_{j}} T_{\alpha}^{(m) g} \mathcal{C}_{\alpha, n}^{m}\right)\right)
$$

is decreasing in $n$. To see this, note that the $n^{\text {th }}$ term in this sequence is an average of the form $\frac{1}{2^{n}}\left(\sum_{i=1}^{2^{n}} a_{i}\right)$ where $a_{i+2^{n-1}} \leq a_{i}$, for $i=1,2, \ldots, 2^{n-1}$. Therefore, the above limit equals

$$
\begin{gathered}
\sum_{j=0}^{2^{m}-1} \lim _{n \rightarrow \infty} \frac{1}{2^{n}} \sum_{i=1}^{2^{n}} H\left(Q \mid \mathcal{A}_{\alpha, i}^{m} \vee\left(\bigvee_{g \in A_{j}} T_{\alpha}^{(m) g} \mathcal{C}_{\alpha, n}^{m}\right)\right) \\
=h\left(T_{\alpha}^{(m)}, Q\right)+\sum_{j=1}^{2^{m}-1} \lim _{n \rightarrow \infty} \frac{1}{2^{n}} \sum_{i=1}^{2^{n}} H\left(Q \mid \mathcal{A}_{\alpha, i}^{m} \vee\left(\bigvee_{g \in A_{j}} T_{\alpha}^{(m) g} \mathcal{C}_{\alpha, n}^{m}\right)\right) .
\end{gathered}
$$

(Here we have just extracted the term corresponding to $j=0$ from the first sum.)

Since the summands are the decreasing limits of upper semicontinuous functions of $\alpha$, each of them is upper semicontinuous. However, by Lemma 14, their sum, $2^{m} h\left(T_{\alpha}, Q\right)$, is continuous, so each of them must be continuous as well.

\section{REFERENCES}

1. Adam Fieldsteel, N. A. Friedman, Restricted orbit changes of ergodic $\mathbf{Z}^{d}$-actions to achieve mixing and completely positive entropy, Ergodic Th. and Dyn. Sys., v. 6 (1986), p. 505-528 MR 88d:28026

2. J. Roberto Hasfura-Buenaga, Mixing for dyadic equivalence, Acta Math. Univ. Comenian, v. LXIV (1995), p. 141-152 MR 97b:28021

3. Andrés del Junco, D. J. Rudolph, Residual behavior of induced maps, Israel J. Math. 93 (1996), 387-398 MR 97d:28022

4. Janet Kammeyer, D. J. Rudolph, Restricted orbit equivalence for ergodic $\mathbf{Z}^{d}$-actions, submitted, Ergodic Th. and Dyn. Sys.

5. Robert R. Phelps, Lectures on Choquet's Theorem, Van Nostrand mathematical studies \#7, Van Nostrand, New York, 1966 MR 33:1690

6. D. J. Rudolph, Restricted orbit equivalence, Mem. Amer. Math. Soc., v. 54 (1985), no. 323 MR 86f: 28021

7. D. S. Ornstein, M. Smorodinsky, Ergodic flows of positive entropy can be time changed to become K-flows, Isr. J. Math., v. 26 (1977), p. 75-83 MR 56:5837

8. D. S. Ornstein, B. Weiss, Every transformation is bilaterally deterministic, Isr. J. Math., vol. 21 (1975), p. 154-158 MR 52:3482

9. D. S. Ornstein, B. Weiss, The Shannon-MacMillan-Breiman theorem for a class of amenable groups, Isr. J. Math., v. 44 (1983), p. 53-60 MR 85f:28018

10. M. Smorodinsky, Ergodic Theory, Entropy, Lect. Notes in Math., vol. 214 (1970) MR 54:10568

11. Klaus Schmidt, Cocycles on ergodic transformation groups, MacMillan lectures in mathematics \#1, MacMillan, Delhi, 1977 MR 58:28262

12. A. M. Stepin, On entropy invariants of decreasing sequences of measurable partitions, Functional Analysis and its Appl., vol. 5 (1971), 237-240 MR 44:1793 
13. J.-P. Thouvenot, Quelques propriétés des systèmes dynamiques qui se décomposent en un produit de deux systèmes dont l'un est un scheme de Bernoulli, Isr, J. Math., v. 21 (1975), p. 177-203 MR 53:3263

14. A. M. Vershik, Decreasing sequences of measurable partitions and their applications, Dokl. Akad. Nauk SSSR, Tom 193 (1970), 748-751; English transl., Soviet Math. Dokl. 11 (1990), 1007-1011 MR 42:3258

15. A. M. Vershik, Continuum of pairwise nonisomorphic diadic sequences, Functional Analysis and its Appl., vol. 5 (1971), 182-184 MR 43:7593

Department of Mathematics, Wesleyan University, Middletown, Connecticut 06459

E-mail address: afieldsteel@mail.wesleyan.edu

Department of Mathematics, Trinity University, San Antonio, Texas 78212

E-mail address: jhasfura@mail.trinity.edu 